\title{
PINK1 Kinase Enhances the Repair Effects of Bone Marrow Mesenchymal Stem Cells on Renal Ischemia/Reperfusion-Induced Acute Kidney Injury in Mice
}

\section{Chenyu Lin}

Beijing Key Laboratory of Immunology Regulatory and Organ Transplantation; Department of Respiratory and Critical Care Medicine Institute the 8th Medical Center, Chinese PLA General Hospital; College of Basic Medicine, Hebei North University

\section{Wen Chen}

Beijing Key Laboratory of Immunology Regulatory and Organ Transplantation; Department of Respiratory and Critical Care Medicine Institute, the 8th Medical Center, Chinese PLA General Hospital Yong Han

Beijing Key Laboratory of Immunology Regulatory and Organ Transplantation; Department of Respiratory and Critical Care Medicine Institute, the 8th Medical Center, Chinese PLA General Hospital Yujie Sun

Beijing Key Laboratory of Immunology Regulatory and Organ Transplantation; Department of Respiratory and Critical Care Medicine Institute, the 8th Medical Center, Chinese PLA General Hospital

\section{Xiaoqiong Zhao}

Beijing Key Laboratory of Immunology Regulatory and Organ Transplantation; Department of Respiratory and Critical Care Medicine Institute, the 8th Medical Center, Chinese PLA General Hospital; Jiamusi University

\section{Yuan Yue}

Beijing Key Laboratory of Immunology Regulatory and Organ Transplantation; Department of Respiratory and Critical Care Medicine Institute, the 8th Medical Center, Chinese PLA General Hospital; Jiamusi University

\section{Binyu Li}

Beijing Key Laboratory of Immunology Regulatory and Organ Transplantation; Department of Respiratory and Critical Care Medicine Institute, the 8th Medical Center, Chinese PLA General Hospital

\section{Wenmei Fan}

Beijing Key Laboratory of Immunology Regulatory and Organ Transplantation; Department of Respiratory and Critical Care Medicine Institute, the 8th Medical Center, Chinese PLA General Hospital

\section{Tao Zhang}

Jiamusi University

Li Xiao ( $\nabla$ xiaolilab309@163.com) 
Beijing Key Laboratory of Immunology Regulatory and Organ Transplantation; Department of Respiratory and Critical Care Medicine Institute, the 8th Medical Center, Chinese PLA General Hospital https://orcid.org/0000-0002-8929-8047

\section{Research Article}

Keywords: PINK1, bone marrow mesenchymal stem cell, ischemia-reperfusion injury, acute kidney injury, mitophagy.

Posted Date: January 12th, 2022

DOI: https://doi.org/10.21203/rs.3.rs-1242192/v1

License: (c) (1) This work is licensed under a Creative Commons Attribution 4.0 International License. Read Full License 


\section{Abstract}

Background: Acute kidney injury (AKI) is a common severe acute syndrome caused by multiple causes, which is characterized by a rapid decline of renal function in a short period. Bone mesenchymal stem cells (BMSCs) are effective in the treatment of AKI. However, it remains unclear about the mechanism of their beneficial effects. PENT-induced kinase 1 (PINK1) may play an important role in the kidney tissue repair. In this study, an endeavor would be made to explore the enhancing effect of PINK1 overexpression on the repair of AKI through BMSCs.

Methods: In this study, the ischemia/reperfusion-induced acute kidney injury (IRI-AKI) in mice and the hypoxia-reoxygenation model of cells were established, and the indexes were detected by pathology and immunology experimental.

Results: After ischemia/reperfusion, compared with the BMSCs group, the OE PINK1 group had a decreased expression of BUN, the mitigated renal fibrosis, the reduced tissue damage degree. Overexpressed PINK1 could decrease the inflammatory reaction of injured kidney tissues in IRI-AKI mice, the decreased expression of IL-10 in peripheral blood serum; and regulate the distribution of immune cells in the kidney during IRI, the decreased infiltration of lymphocytes, the increased infiltration of macrophages; and reduce the stress response of BMSCs under hypoxia and inflammation; and enhance the stress response of BMSCs to renal tubular epithelial cells(RTECs) under hypoxia and inflammation, the decreased apoptosis rate of RTECs, the decreased release of TNF- $\alpha$ in the cell supernatant, and the decreased proliferation of PBMCs in peripheral blood after hypoxia and reoxygenation; and regulate the autophagy of BMSCs in kidney tissues with IRI-AKI to better repair the injured kidney tissues, the increased expression of LC3-B related to autophagy and the decreased expression of mTOR.

Conclusions: In this study, PINK1 overexpression enhances the repair effect of BMSCs on IRI-AKI, and the distribution of injured renal immune cells during IRI regulation by BMSCs. Besides, PINK1 enhances BMSCs and their resistance to the stress response of RTECs under hypoxia and inflammation. In addition, it regulates mitophagy during IRI-AKI. The findings of this study provide a new direction and target for the repair of IRI-AKI through BMSCs.

\section{Introduction}

Acute kidney injury (AKI) is a severe acute syndrome caused by multiple causes, which is characterized by a rapid decline of renal function. It has become one of the serious diseases affecting human health and longevity due to its high incidence, high mortality and poor prognosis $[1,2]$. The leading causes of AKI include sepsis, nephrotoxin, renal ischemia-reperfusion injury (IRI) and so forth [3]. IRI is the main reason of endogenous AKI. In clinical practice, hypovolemia, hypotension or heart failure can cause transient ischemia, which will further induce AKI; Besides, IRI would affect renal transplantation and other operations that require temporary blood supply interruption. Acute tubular necrosis caused by IRI, especially the early graft dysfunction and immunogenicity enhancement caused by IRI during kidney 
transplant, would seriously affect the prognosis of patients [4]. There is a lack of effective treatment for AKI. The symptomatic treatment, supportive treatment or renal replacement therapy is usually adopted in clinical practice through getting rid of etiology and correcting reversible risk factors. Currently, due to the fact that there is no specific treatment for $\mathrm{AKI}$ at home and abroad, kidney injury cannot be alleviated, the normal repair of injured cells cannot be promoted, and pathological repair cannot be inhibited. A more thorough understanding of the basic molecular and immunological mechanisms underlying this complex injury conduces to identifying an innovative starting point for therapeutic intervention.

The pathophysiological process of renal ischemia/reperfusion-induced acute kidney injury (IRI-AKI) is complex. SubstAntial evidence demonstrate that mitophagy is the key link in the development of AKI. IRIinduced AKI is closely related to changes in mitochondrial function. Injured mitochondria can trigger apoptosis of renal tubular cells, activate NOD-like receptor protein 3 (NLRP3) inflammasome, and eventually cause renal function loss [5]. One of the key adaptive responses of mitochondria to various stress injuries is to activate mitophagy. During this process, injured mitochondria would be rapidly and selectively removed in an autophagy-dependent manner, thus reducing the excessive release of reactive oxygen species (ROSs) and pro-apoptosis substances caused by injured mitochondria. This mechanism is closely related to apoptosis, tissue remodeling and fibrosis. Therefore, the maintenance of mitochondrial homeostasis and function through promoting mitophagy may improve ischemic kidney injury [6]. Mitophagy is mainly regulated by ubiquitin-dependent and -independent mechanisms, among which the ubiquitin-dependent pathway is PTEN induced putative kinase 1 (PINK1)/Parkin pathway. Recent reports suggest that PINK1/Parkin pathway plays an important role in AKI mitochondrial quality control, renal tubular cell survival and renal function protection [7]. PINK1 is the key enzyme of mitophagy, and it has protein kinase activity and can regulate cell function by phosphorylating various proteins in cells [8]; Besides, this enzyme can regulate mitochondrial morphology, function and autophagy through multiple pathways; it has an extremely low expression level when mitochondrial function is normal, but a high expression level in high energy consumption tissues; it also has various biological activities, which may participate in the occurrence, development and regulation of various diseases [9]. Kidney tissues with high energy consumption have abundant mitochondria, and mitochondrial damage or dysfunction is closely related to AKI caused by various reasons. Therefore, PINK1 kinase is a potential target site for the accurate treatment of AKI.

In recent years, stem cell therapy has become one of the most promising treatments for renal IRI-AKI, with greater potential value than single drug therapy due to the highly diverse responses of cells to the environment. Importantly, bone marrow mesenchymal stem cells (BMSCs) have physiological activities in interstitial fibrosis, inflammation, angiogenesis and immune regulation. According to many studies, BMSCs are effective in various preclinical models of kidney injury, or may improve the structure and function of the kidney after IRI by reducing inflammation and apoptosis, promoting autophagy, improving tissue fibrosis and accelerating the proliferation of renal interstitial cells through paracrine and endocrine factors $[10,11]$. Although BMSCs have high therapeutic potential for kidney injuries, the transplantation and therapeutic effects of these cells are limited by pathophysiological conditions of the injured site. Glucose deficiency, oxidative stress, ischemia and inflammation would induce allograft dysfunction, 
affect the survival of transplanted BMSCs, and result in the death of transplanted cells in injured tissues $[12,13]$. Among them, oxidative stress is the most unfavorable factor. As is revealed in some studies, mitochondrial dysfunction reduces the potential of proliferation, migration and pluripotency of BMSCs $[14,15]$. Therefore, in order to improve the repair effect of BMSCs, it is of great significance to improve the survival rate of BMSCs transplanted to the injured site by improving the resistance of BMSCs under those pathophysiological conditions.

As a key enzyme of mitophagy, PINK1 was induced in this study by gene transfection, and overexpressed in BMSCs to resist the damage to BMSCs under IRI-AKI conditions, which exerted active impacts on the renal protection by improving the survival and differentiation of BMSCs under IRI-AKI conditions and enhancing mitophagy, thus enhancing the repair effect of BMSCs on IRI-AKI.

\section{Materials And Methods}

\section{Animals}

Male C57BL/6 mice (6-8 week old, SPF grade) were purchased from SPF (Beijing) Biotechnology Co., Ltd., with the animal license No. being SCXK (Jing) 2019-0010. All experiments were approved by the Ethics Committee of Laboratory Animal Management in the Eighth Medical Center of Chinese PLA General Hospital.

\section{Cell Culture}

Bone marrow mesenchymal stem cells (BMSCs) of C57BL/6 mice and red fluorescent protein labeled bone marrow mesenchymal stem cells (RFP-BMSCs) (cyagen; MUBMX-01001, MUBMX-01201) were subjected to routine culture. After $24 \mathrm{~h}$ of resuscitation, the medium was changed for the first time, and the culture medium was changed every $2-3 \mathrm{~d}$. $80 \%-90 \%$ cells were passaged at fusion. The cells of the third generation were digested with $0.25 \%$ trypsin (Bl; Israel) containing $0.02 \%$ EDTA, washed twice with PBS (Hyclone; USA), and then resuspended with PBS to make living cell suspension with the concentration of $1 \times 10^{7}$ cells $/ \mathrm{mL}$.

Renal tubular epithelial cells (RTECs) of C57BL/ 6 mice (Procell; CP-M062) were recovered in a $25 \mathrm{~mL}$ gelatin-coated culture flask with C57BL/6 Mice RTECs Complete Medium (Procell; CM-M062) in a water bath to $37^{\circ} \mathrm{C}$ and placed in the incubator for routine incubation. The third generation cells were resuspended with PBS and made into living cell suspension, with the concentration of $1 \times 10^{7}$ cells $/ \mathrm{mL}$.

\section{Overexpression of PINK1 in BMSCs}

After the digestion and centrifugation of $80 \%-90 \%$ of the fused cells, these cells were precipitated and suspended to the required concentration with the medium without double Antibody, and inoculated into a 12-well plate (Corning; USA) at a concentration of $0.5 \times 10^{4} \mathrm{cell} / \mathrm{mL}$; Subsequently, the plate was routinely cultured at $37^{\circ} \mathrm{C}, 5 \% \mathrm{CO}_{2}$ and $95 \%$ humidity, and the medium without double Antibody was replaced when $60 \%-70 \%$ fusion was completed. After adding the buffer, plasmid and transfection reagent in 
proportion to the working solution according to the instructions of jetPRIME Transfection Kit (Polyplus Transfection; 23Y2307M8) for 4h, the medium was replaced with fresh medium without double Antibody and continued to incubate for $24 \mathrm{~h}$. The transfection efficiency was observed by fluorescence microscopy and detected by flow cytometry for further experiments.

\section{Neutralization and closure of PINK1 in BMSCs}

BMSCs were inoculated in a 12-well plate at a concentration of $0.5 \times 10^{4} \mathrm{cell} / \mathrm{mL}$. When $60 \%-70 \%$ fusion was completed, the medium was replaced with fresh medium and 10uL Anti-PINK1 (Abcam; ab23707) was added. Subsequently, the culture was continued for $24 \mathrm{~h}$ to neutralize and close PINK1 on the surface of BMSCs.

\section{Establishment of Animal Kidney IRI Model}

Mice were randomly divided into 5 groups: (1) sham operation control (sham) group $(n=6)$; (2) renal ischemia/reperfusion injury (IRI) group $(n=6)$; (3) untreated bone marrow mesenchymal stem cell therapy (BMSCs) group ( $n=6)$; (4) BMSCs with overexpressed PINK1 (OE PINK1) group $(n=6)$; (5) BMSCs with neutralized PINK1 (Anti PINK1) group $(n=6)$.

In the sham group, mice were anesthetized with $2 \%$ pentobarbital $(40 \mathrm{mg} / \mathrm{kg})$ and fixed on a constant temperature plate at $37^{\circ} \mathrm{C}$. The midline abdominal incision was performed, and the skin, subcutaneous tissue and peritoneum were separated layer by layer. Subsequently, the abdomen was covered with gauze soaked in normal saline for $30 \mathrm{~min}$ and then sutured; In the IRI group, the procedure was the same as the control group. After opening the abdominal cavity, the left renal pedicle was found first and then quickly clipped with a non-invasive artery clamp, followed by timing. Then, the right renal pedicle clip was found, followed by timing. The arterial clip was removed after $30 \mathrm{~min}$ to realize the perfusion with blood flow, and the abdomen was subsequently sutured 15; In the BMSCs Group, IRI-AKI model was established in the same way as IRI Group, and $0.1 \mathrm{ml}$ of BMSCs cell suspension $\left(1 \times 10^{7}\right.$ cells $\left./ \mathrm{ml}\right)$ was infused through the caudal vein; In the OE PINK1 group, the procedure was the same as the BMSCs group. $0.1 \mathrm{~mL}$ of BMSCs cell (with overexpressed PINK1) suspension $\left(1 \times 10^{7} \mathrm{cells} / \mathrm{mL}\right)$ was infused through the caudal vein; In the Anti PINK1 group, the procedure was the same as the BMSCs group. $0.1 \mathrm{~mL}$ of BMSCs cell (with neutralized PINK1) suspension $\left(1 \times 10^{7}\right.$ cells $\left./ \mathrm{mL}\right)$ was infused through the caudal vein.

\section{Renal Function Analysis}

After the IRI-AKI model of mice was established successfully, the state of mice in each group was observed every $24 \mathrm{~h}$. After $72 \mathrm{~h}$, the blood was collected from the mouse eyes of each group and numbered. After being placed at room temperature for $30 \mathrm{~min}$ to present obvious stratification, the supernatant was centrifuged at $3500 \mathrm{r} / \mathrm{min}$ for $5 \mathrm{~min}$. The serum was aspirated for the determination of serum creatinine (SCR) and blood urea nitrogen (BUN) on an automatic biochemical analyzer (Hitachi 7180; Japan).

\section{Histopathology}


Kidney samples of mice were collected and fixed with formalin. These kidney tissues were fixed with paraffin embedment, and the continuous section was performed, with $4 \mu \mathrm{m}$ in thickness, followed by routine hematoxylin-eosin (HE) staining. An optical microscope (Nikon; Japan) was adopted for the observation. Stained tissue sections were scored with a semi-quAntitative scale, in which a double-blind method was employed to assess the degree of tubular necrosis. The renal tubular injury was characterized by loss of brush border, dilatation and/or atrophy of renal tubules, degeneration of renal tubules, formation and vacuolation of renal tubules, infiltration of inflammatory cells or edema of cells, with scores ranging from 0 to 4 . The higher the score, the more serious the injury, which can be specifically manifested as $0=$ normal kidney, $1=$ mild necrosis (the degree of cortex or medulla involvement $<5 \%$ ), $2=$ mild to moderate necrosis (the degree of cortex or medulla involvement $5-25 \%$ ), $3=$ moderate necrosis (the degree of cortex or medulla involvement 25-75\%), 4=severe necrosis (the degree of cortex or medulla involvement $>75 \%$ ).

\section{Immunohistochemistry}

The prepared tissue slices with paraffin embedment (thickness 3-4 $\mu \mathrm{m}$ ) were dewaxed, rehydrated by continuous immersion in ethanol, and sealed with peroxidase sealing solution for $5 \mathrm{~min}$. The primary Antibodies (Anti-PINK1, ab23707; Anti-LC3, ab48394; Anti-mTOR, ab134903; Anti-CD3, ab135372; AntiCD14, ab221678; Anti-CD20, ab64088; Anti-CD68, ab125212; 1:250, Abcam) were incubated with the sections and then stained with biotin-linked secondary Antibodies before the treatment with streptavidin (DACCO Cytomation, K3461). Subsequently, the glass slide was treated with AEC solution at room temperature for $10 \mathrm{~min}$ and images were taken with a fluorescence microscope (Nikon; Japan).

\section{Terminal deoxynucleotidyl transferase dUTP nick end labeling (TUNEL) staining}

The TUNEL reaction mixture was prepared with the In Situ Cell Death Test Kit (Roche; 11684817910) according to the instructions and placed on an ice box for later use. After routine dewaxing and rehydration, the glass slide was placed into $200 \mathrm{~mL}$ of citrate buffer with $0.1 \mathrm{M} \mathrm{PH}=6.0$, irradiated with 350W microwave for $5 \mathrm{~min}$. Subsequently, the glass slide was washed twice with PBS for $5 \mathrm{~min}$ each time.

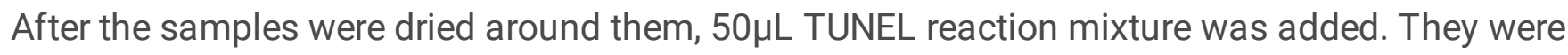
incubated in a constant temperature incubator (Shanghai Sumsung Laboratory Instrument Co., Ltd) for $60 \mathrm{~min}$ in a humid and dark environment at $37^{\circ} \mathrm{C}$. After being washed with PBS for 3 times, they were sealed with an Antifading medium with DAPI (ZSGB-BIO; ZLI-9557), and analyzed under a fluorescence microscope. Apoptosis of mouse kidney tissues was observed under a fluorescence microscope with a magnification of 400X. The number of TUNEL-positive renal tissue cells in at least 10 light fields in each tissue section of 3 different kidneys in each group was quAntitatively calculated. The number of TUNELpositive renal tissue cells in each square millimeter of the kidney tissue section was calculated.

\section{Establishment of BMSCs Targeting Model for Injured Kidney Tissues}


The left kidney ischemia-reperfusion model was established as described in Section 2.5. 0.1 mL of RFPBMSCs cell suspension ( $\left.1 \times 10^{7} \mathrm{cells} / \mathrm{ml}\right)$ was infused into the caudal vein. These mice were subjected to humanitarian euthanasia after $24 \mathrm{~h}$, and fresh bilateral kidney tissues were taken and frozen to prepare sections. The homing of BMSCs in bilateral tissues was observed through a fluorescence microscope.

\section{Establishment of Hypoxia-reoxygenation Model of Cells}

After the passaged C57BL/ 6 mouse BMSCs were fused with RTECs to about $50 \%$, serum starvation was continued for $24 \mathrm{~h}$. Subsequently, the cell samples were washed with PBS twice, immersed in mineral oil (Sigma-Aldrich; M5310) at $37^{\circ} \mathrm{C}$ for $60 \mathrm{~min}$, washed with a large amount of PBS for many times, and cultured in the complete culture medium for 24h [16].

\section{Co-culture of Cells}

Normal C57BL/6 mouse BMSCs were co-cultured with hypoxia-reoxygenation C57BL/6 mouse RTECs under conventional conditions (1:10); Normal C57BL/ 6 mouse BMSCs were co-cultured with normal C57BL/ 6 mouse RTECs under conventional conditions (1:10). After $60 \%-80 \%$ fusion was completed, they were treated under the hypoxia-reoxygenation conditions.

\section{Flow Cytometry(FCM) analysis Apoptosis}

Cell preparation was based on PE Annexin V Apoptosis Detection Kit I (BD, No. 559763). The cells were digested with trypsin without EDTA (Bl; Israel). After the collection of cells, they were washed twice with precooled PBS at $4^{\circ} \mathrm{C}$. The concentration of the cells was adjusted to $1 \times 10^{6} \mathrm{cell} / \mathrm{ml}$ by adding $1 \mathrm{x}$ buffer. The single cell suspension of $1 \times 10^{5}$ cell/tube was taken and added with $5 \mu \mathrm{L} /$ tube PE Annexin V. After being mixed, these cells were incubated in darkness at room temperature for $15 \mathrm{~min}$. Subsequently, $5 \mu \mathrm{L} /$ tube 7-AAD and proper buffer were added into each flow tube. FCM analysis was performed by flow cytometry (BD; USA) within $1 \mathrm{~h}$.

\section{Cell Proliferation Test}

Cell suspension (100uL) was arranged in a 96-well plate. The culture plate was pre-cultured in an incubator for $24 \mathrm{~h}\left(37^{\circ} \mathrm{C}, 5 \% \mathrm{CO}_{2}\right)$. $10 \mathrm{uL}$ of $\mathrm{CCK}$ solution was added to each well, and the formation of bubbles in the well was avoided. The culture plate was incubated in the incubator for $3 \mathrm{~h}$. The absorbance at $450 \mathrm{~nm}$ was measured by a microplate reader.

\section{Enzyme-linked Immunosorbent Assay}

The contents of interleukin-10 (IL-10) and tumor necrosis factor-a (TNF-a) in serum and cell supernatant were detected by enzyme-linked immunosorbent assay (ELISA) (ELISA kit: Neobioscience; EMC102a.96, EMC005.96). The experimental steps were conducted according to the instructions, and OD450 was measured immediately after completion (within $3 \mathrm{~min}$ ). The data were saved.

\section{Statistical Methods}


All statistical analysis was conducted with SPSS 23.0 software. According to the measurement data of normal distribution, the t-test or one-way ANOVA was adopted to analyze the comparison of mean between groups, and the results were expressed as mean \pm standard deviation. Among them,independent sample t-test was adopted for the comparison between both groups; one-way ANOVA was adopted for the analysis of the difference among multiple groups; LSD test was adopted for pairwise comparison between groups; and Dunnett's T3 test was adopted for the comparison between groups with a heterogeneity of variance; Kruskal-Wallis $\mathrm{H}$ test was adopted for the comparison of non-normal distribution data and ranked data, and the results were expressed as the median value (interquartile range). $\mathrm{P}<0.05$ can be considered to be statistically significant.

\section{Results}

PINK1 Participated in the Protective Effect of BMSCs on Kidney Tissues with IRI-AKI.

PINK1 was closely related to the repair of kidney tissues by BMSCs in IRI-AKI mice

Firstly, the pathological changes of kidney tissues in IRI-AKI mouse model were subjected to an observation. It was demonstrated in the histological examination of HE staining that the kidney structure of mice in the sham group was normal under physiological conditions, while the renal tubular injury was obvious in the mouse kidney of the IRI group. Among the three BMSCs intervention control groups (the BMSCs group, the OE PINK1 group and the Anti PINK1 group), the OE PINK1 group had the most moderate kidney injury, followed by the IRI group, and the Anti PINK1 group had the most serious kidney injury (Figure 1a). Among them, based on the kidney injury score, the degree of renal tubular injury in the OE PINK1 group $(1,1)$ was significantly lower than that in the IRI group $(3,1)(P<0.001)$ and the Anti PINK1 group $(4,1)(P<0.001)$, and there was no significant difference between the OE PINK1 group and the sham group $(0,1)(P=0.167)$, and the OE PINK1 group was lower than the BMSCs group $(2,1)(P=0.056)$, but there was no statistical difference as well(Figure 1b). Consistent with the results of histological analysis, the OE PINK1 group had a lower concentration of SCR $(11.22 \pm 1.48 \mu \mathrm{mol} / \mathrm{L})$ and BUN $(11.57 \pm 0.83 \mathrm{mmol} / \mathrm{L})$, and there was a significant difference between the OE PINK1 group and the IRI group (SCR: $25.45 \pm 3.10 \mu \mathrm{mol} / \mathrm{L}, \mathrm{BUN}$ : $21.61 \pm 2.08 \mathrm{mmol} / \mathrm{L})(P<0.001 ; P<0.001)$. There was also a significant difference in the expression level of BUN between the OE PINK1 group and the BMSCs group $(17.10 \pm 1.69 \mathrm{mmol} / \mathrm{L})(P<0.05)$; While, the concentrations of SCR and BUN in the IRI group and the Anti PINK1 group (SCR: $22.24 \pm 4.36 \mu \mathrm{mol} / \mathrm{L}, \mathrm{BUN}$ : $20.08 \pm 5.21 \mathrm{mmol} / \mathrm{L}$ ) were significantly increased (Figure $1 \mathrm{C}$ and d). These results indicated that renal dysfunction occurred in mice with IRI, BMSCs could improve renal function and repair injured kidney tissues; Besides, overexpressed PINK1 could enhance the repair effect of BMSCs, and neutralized PINK1 could make BMSCs lose its repair effect.

Besides, The characteristics of BMSCs targeting injured kidney tissues were verified by infusing BMSCs with fluorescent labels into the mouse caudal vein in the unilateral kidney injury model and observing the number of fluorescent cells in injured and normal kidneys of the same mouse, as shown in Figure $1 \mathrm{f}$. It 
was found that the number of fluorescent cells on the injured side was relatively higher $(83.75 \pm 26.26$ $\left./ \mathrm{mm}^{2}\right)$, with a significant difference compared with the control side $\left(8.75 \pm 8.5 / \mathrm{mm}^{2}, t=5.432, P=0.002\right)$, as shown in Figure 1g. These results indicated that BMSCs could target injured tissues.

PINK1 was closely related to the inhibiting effect of BMSCs on apoptosis in kidney tissues of IRI-AKI mice.

TdT-mediated dUTP nick-end labeling (TUNEL) was adopted to detect apoptosis in kidney tissues of mice, and the effect of overexpressed and neutralized PINK1 on apoptosis of kidney tissues was subjected to an observation. As shown in Figure 2, the OE PINK1 group had the least number of TUNEL-positive cells in kidney tissues $\left(41.5 \pm 17.93 / \mathrm{mm}^{2}\right)$, while the IRI group $\left(156.87 \pm 26.38 / \mathrm{mm}^{2}\right)$ and the Anti PINK1 group $\left(194.22 \pm 67.79 / \mathrm{mm}^{2}\right)$ had an increased number of these cells. These results indicated that the apoptotic cells in the BMSCs group $\left(86.32 \pm 18.44 / \mathrm{mm}^{2}\right)$ were significantly lower than those in the IRI group $(P<0.001)$, and the TUNEL-positive cells in kidney tissues in the OE PINK1 group and the Anti PINK1 group were significantly different from those in the BMSCs group $(P<0.001, P<0.001)$ (Figure $2 b)$. The results suggested that BMSCs could play a protective role in the kidney tissues of IRI-AKI mice and reduce the apoptosis induced by IRI. Besides, overexpressed PINK1 overexpression could enhance the effect of BMSCs on the apoptosis of injured tissues, while neutralized PINK1 could weaken the protective effect of BMSCs.

PINK1 participated in the alleviating effects of BMSCs on the inflammatory reaction of kidney tissues in IRI-AKI mice.

Inflammation plays an important role in the occurrence and progression of IRI-AKI. Therefore, renal inflammation was evaluated by observing the infiltration of renal immune cells (macrophages, monocytes, T cells, B cells and so forth) by immunohistochemical staining, as shown in Figure 3a. In terms of the expression of $\mathrm{CD}^{+}$, the IRI group $\left(170.98 \pm 31.39 / \mathrm{mm}^{2}\right)$ had a significantly higher level than the sham group $\left(49.80 \pm 22.73 / \mathrm{mm}^{2}\right)(P<0.001)$; the BMSCs group $\left(89.64 \pm 23.03 / \mathrm{mm}^{2}\right)(P<0.001)$ and the OE PINK1 group $\left(43.16 \pm 25.85 / \mathrm{mm}^{2}\right)(P<0.001)$ had a relatively lower level than the IRI group; the OE PINK1 group had a relatively lower level than the BMSCs group $(P=0.01)$ and the Anti PINK1 group $\left(91.30 \pm 24.20 / \mathrm{mm}^{2}\right)(P=0.008)$, as shown in Figure $3 \mathrm{~b}$. In terms of the expression of $C D 14^{+}$, the IRI group $\left(605.90 \pm 59.56 / \mathrm{mm}^{2}\right)$ had a significantly higher level than the sham group $\left(317.06 \pm 54.55 / \mathrm{mm}^{2}\right)$ $(P<0.001)$; the OE PINK1 group $\left(873.16 \pm 105.87 / \mathrm{mm}^{2}\right)$ had a relatively higher level than the IRI group $(P=0.017)$ and the Anti PINK1 group $\left(609.22 \pm 37.40 / \mathrm{mm}^{2}\right)(P=0.023)$, and the OE PINK1 group had a higher level than the BMSCs group $\left(715.46 \pm 73.87 / \mathrm{mm}^{2}\right)$, but the difference was not statistically significant $(P=0.192)$, as shown in Figure $3 c$. There was a similar changing trend in the expression level of $\mathrm{CD}_{2} \mathrm{O}^{+}$and $\mathrm{CD}^{+}$, and the differences were not significant. Compared with the IRI group $(64.74 \pm 12.31$ $\left./ \mathrm{mm}^{2}\right)$, the sham group $\left(34.86 \pm 18.93 / \mathrm{mm}^{2}\right)(P=0.01)$ and the OE PINK1 group $\left(38.18 \pm 19.11 / \mathrm{mm}^{2}\right)$ $(P=0.019)$ had a significantly lower level in their expression; the Anti PINK1 group $\left(69.72 \pm 19.11 / \mathrm{mm}^{2}\right)$ had a slightly higher level than the BMSCs group $\left(44.82 \pm 11.14 / \mathrm{mm}^{2}\right)(P=0.027)$ and the OE PINK1 group $(P=0.007)$; the OE PINK1 group had a lower level than the BMSCs group, but the difference was not 
statistically significant $(P=0.532)$, as shown in Figure $3 d$. In terms of the expression of $\mathrm{CD}^{2} 8^{+}$, the IRI group $\left(175.96 \pm 31.28 / \mathrm{mm}^{2}\right)$ had a significantly higher level than the sham group $\left(79.68 \pm 27.28 / \mathrm{mm}^{2}\right)$ $(P=0.007)$ and the BMSCs group $\left(81.34 \pm 24.48 / \mathrm{mm}^{2}\right)(P=0.007)$, and had a significantly lower level than the OE PINK1 group $\left(640.76 \pm 86.73 / \mathrm{mm}^{2}\right)(P<0.001)$; the OE PINK1 group had a relatively higher level than the BMSCs group $(P<0.001)$ and the Anti PINK1 group $\left(179.28 \pm 42.56 / \mathrm{mm}^{2}\right)(P<0.001)$, as shown in Figure $3 e$. The above results demonstrated that IRI could induce the infiltration of lymphocytes, macrophages, monocytes and B cells in injured tissues, while BMSCs infusion could reduce the infiltration of lymphocytes, B cells and macrophages and increase the infiltration of monocytes; PINK1 enhanced the promoting effect of BMSCs on decreasing the infiltration of lymphocytes and B cells, and increased the infiltration of macrophages and monocytes; PINK1 neutralization inhibited this effect. Moreover, the expression of inflammatory cytokines interleukin-10 (IL-10) and tumor necrosis factor-a (TNF-a) in peripheral blood serum of IRI-AKI mice was analyzed by ELISA. The results showed that compared with the sham group(IL-10: $120.27 \pm 11.81 \mathrm{pg} / \mathrm{ml}$; TNF-a: $80.90 \pm 10.90 \mathrm{pg} / \mathrm{ml}$ ), the IRI group had an increased expression level of IL-10 $(549.42 \pm 12.58 \mathrm{pg} / \mathrm{ml}, \mathrm{P}<0.001)$ and TNF-a $(116.69 \pm 13.06$ $\mathrm{pg} / \mathrm{ml}, \mathrm{P}=0.01$ ); the BMSCs group (IL-10: $175.54 \pm 11.69 \mathrm{pg} / \mathrm{ml}, \mathrm{P}<0.001 ;$ TNF-a: $45.70 \pm 10.60 \mathrm{pg} / \mathrm{ml}$, $\mathrm{P}<0.001$ ) had a significantly decreased level compared with the IRI group; The OE PINK1 group $(73.32 \pm 11.72 \mathrm{pg} / \mathrm{ml}, \mathrm{P}<0.001)$ had a lower expression level of IL-10 compared with the BMSCs group, and there was no significant difference in the expression level of TNF-a $(58.14 \pm 10.60 \mathrm{pg} / \mathrm{ml}, \mathrm{P}=0.176)$ between both groups; The Anti PINK1 group (IL-10: $575.38 \pm 11.18$ pg/ml, $P<0.001$; TNF-a: $221.70 \pm 14.30$ $\mathrm{pg} / \mathrm{ml}, \mathrm{P}<0.001$ ) had a higher expression level of both inflammatory cytokines than the BMSCs group,as shown in Figure $3 \mathrm{f}$ and $\mathrm{g}$. These results indicated that BMSCs could alleviate the inflammatory reaction of kidney tissues in IRI-AKI mice, and PINK1 can further enhance the Anti-inflammatory effect of BMSCs; These results suggested that the Anti-inflammatory effect of BMSCs on IRI-AKI enhanced by PINK1 may be related to macrophages and monocytes.

Stress Response of BMSCs under Hypoxia-reoxygenation Conditions

PINK1 participated in the apoptosis of BMSCs under hypoxia-reoxygenation conditions.

The in vitro hypoxia-reoxygenation model was established to simulate the in vivo IRI environment. FCM method was adopted to evaluate the apoptosis of BMSCs, overexpressed PINK1-BMSCs and neutralized PINK1-BMSCs under hypoxia-reoxygenation conditions, as shown in Figure 4a. Compared with the control group $(13.50 \pm 0.58)$, the IRI group $(21.50 \pm 2.08, P<0.001)$ had a significantly increased apoptosis rate, the OE PINK1 group $(8.05 \pm 1.07, \mathrm{P}<0.001)$ had a significantly decreased apoptosis rate than the IRI group, and the Anti PINK1 group $(18.00 \pm 0.53, \mathrm{P}=0.002)$ also had a decreased apoptosis rate compared with the IRI group, as shown in Figure $4 \mathrm{~b}$. These results indicated that the apoptosis rate of BMSCs increased under hypoxia stress, and PINK1 could reduce the apoptosis of BMSCs under hypoxia-reoxygenation conditions. 
PINK1 participated in the inflammatory response of BMSCs under hypoxia-reoxygenation conditions.

In this study, the in vitro hypoxia-reoxygenation model was established to simulate the in vivo IRI environment. The FCM method was employed to evaluate the expression level of inflammatory factors in BMSCs, overexpressed PINK1-BMSCs and neutralized PINK1-BMSCs under hypoxia-reoxygenation conditions, among which there was no significant change in the expression level of IL-10 $(P=0.834)$. In terms of the expression level of TNF-a, compared with the control group $(52.49 \pm 2.22 \mathrm{pg} / \mathrm{ml})$, the IRI group $(65.94 \pm 8.71 \mathrm{pg} / \mathrm{ml}, \mathrm{P}=0.027)$ had a significantly increased level, the OE PINK1 group $(28.90 \pm 1.76 \mathrm{pg} / \mathrm{ml}$, $\mathrm{P}=0.030)$ had a significantly decreased level, and the Anti PINK1 group $(38.22 \pm 1.98 \mathrm{pg} / \mathrm{ml}, \mathrm{P}<0.01)$ also had a significantly decreased level, as shown in Figure $4 \mathrm{c}$ and $\mathrm{d}$. These results indicated that BMSCs had increased pro-inflammatory factors under hypoxia stress, and PINK1 could reduce the release of proinflammatory factors under hypoxia-reoxygenation conditions.

PINK1 participated in the stress response of BMSCs against RTECs under hypoxia-reoxygenation conditions

PINK1 participated in the effect of BMSCs on reducing apoptosis of RTECs under hypoxia-reoxygenation conditions

In this study, the in vitro hypoxia-reoxygenation model was established to simulate the in vivo IRI environment. The RTECs of three BMSCs intervention control groups (the BMSCs group, the OE PINK1 group and the Anti PINK1 group) were evaluated before and after hypoxia and reoxygenation. Those RTECs co-cultured with BMSCs after ischemia-reperfusion injury were divided into the IRI-Co culture group; while, those co-cultured RTECs before hypoxia and reoxygenation were divided into the Co cultureIRI group. Their effects on apoptosis of RTECs were observed after $24 \mathrm{~h}, 48 \mathrm{~h}$ and $72 \mathrm{~h}$, respectively, as shown in Figure 5a. Among them, Our study found that the apoptosis rate decreased gradually with time, as shown in Figures $5 \mathrm{~b}-\mathrm{d}$. At $24 \mathrm{H}$ and $48 \mathrm{H}$, the apoptosis rate of each group was significantly different from that of the IRI group $(24 \mathrm{H}: 16.33 \pm 0.12,48 \mathrm{H}: 12.93 \pm 0.55)$, regardless of the order of co-culture, the apoptosis rate of the OE PINK1 group was significantly lower than that of the BMSCs group $(P<0.001$, $\mathrm{P}<0.001, \mathrm{P}<0.001, \mathrm{P}<0.001$ )(shown in Fig. 5b-c); the apoptosis rate of the IRI-Co culture-BMSCs group $(11.37 \pm 0.21)$ was significantly lower than that of the Co culture-IRI-BMSCs group $(14.90 \pm 0.56)(P<0.001)$ at $48 \mathrm{~h}$, and the apoptosis rate of the IRI-Co culture-OE PINK1 group (8.77 \pm 0.40$)$ was significantly lower than that of the Co culture-IRI-OE PINK1 group $(11.67 \pm 0.29)(P<0.001)$, as shown in Figures $5 \mathrm{c}$. The results of $48 \mathrm{H}$ showed that BMSCs without hypoxic reoxygenation stimulation may have better antiapoptotic ability; and these results indicated that OE PINK1 can reduce apoptosis induced by hypoxia and reoxygenation and enhance the survival function of BMSCs during the stress response.

Overexpressed PINK1 in vitro enhanced the resistance of BMSCs to the inflammatory response of RTECs

Page 12/29 
under hypoxia-reoxygenation conditions, including changing the release of inflammatory factors and promoting the proliferation of peripheral blood mononuclear cells

Based on the grouping in above-mentioned, the influence of inflammatory factor release in the supernatant of co-culture was observed after $24 \mathrm{~h}, 48 \mathrm{~h}$ and $72 \mathrm{~h}$, respectively, as shown in Figure 6a-f. Among them, there was no significant difference in the release of inflammatory factors after $48 \mathrm{~h}(\mathrm{P}$ $=0.26)$; ELISA analysis revealed that the expression level of IL-10 in the IRI-Co culture group was higher than that in the Co culture group at all three time periods, and the change was significant at $24 \mathrm{~h}$ $(P<0.001)$. The expression level of IL-10 in the IRI-Co culture-OE PINK1 group $(26.16 \pm 2.94 \mathrm{pg} / \mathrm{ml})$ was higher than that in the Co culture-IRI-OE PINK1 group $(19.35 \pm 2.24 \mathrm{pg} / \mathrm{ml})$ after $24 \mathrm{~h}(\mathrm{P}=0.049)$ and the expression level of IL-10 in the OE PINK1 group was significantly lower than that in the Anti PINK1 group regardless of co-culture sequence $(P=0.002 ; P=0.006)$. While, the expression level of TNF-a changed significantly at $72 \mathrm{~h}(\mathrm{P}<0.001)$, the expression level of TNF-a in the Co culture-IRI-OE PINK1 group $(11.11 \pm 2.67 \mathrm{pg} / \mathrm{ml})$ was significantly lower than that in the Co culture-IRI-BMSC group $(20.13 \pm 3.12$ $\mathrm{pg} / \mathrm{ml})(P=0.003)$ and the Co culture-IRI-Anti PINK1 group $(26.33 \pm 2.99 \mathrm{pg} / \mathrm{ml})(P<0.001)$. These results indicated that PINK1 overexpression could reduce the release of inflammatory factors induced by hypoxia and reoxygenation.

Moreover, after extracting peripheral blood mononuclear cells (PBMCs) in mice, the proliferation of PBMCs was evaluated by Cell Counting Kit-8 (CCK8). Normal BMSCs and hypoxia-reoxygenation BMSCs were co-cultured with the PBMCs isolated from normal mice and mice with IRI, respectively. The changes in the proliferation of PBMCs were measured, in an attempt to explore the Anti-inflammatory effect of BMSCs in vivo, as shown in Figure $6 \mathrm{~g}$. It can be observed that the IRI in kidney tissues could cause changes in PBMCs $(P=0.017)$. Compared with the IRI PBMCs, BMSCs $(P=0.088)$ and hypoxiareoxygenation BMSCs $(P=0.059)$ could promote the proliferation of PBMCs, but there were no statistical difference; Anti PINK1-induced BMSCs $(P=0.023 ; P=0.030 ; P=0.007)$ could promote the proliferation of PBMCs; while, overexpressed PINK1-induced BMSCs ( $P=0.206 ; P=0.199 ; P=0.405)$ could not promote the proliferation of PBMCs. These results indicated that there were rapid changes in PBMCs during the response to external oxidative stress; while, BMSCs would generate a series of effects during the response to oxidative stress, and these effects could stimulate the proliferation of PBMCs as well.

PINK1 Participated in IRI-AKI through Targeting Mitophagy.

PINK1 regulated mitophagy, and IRI-AKI was closely related to autophagy. The immunohistochemical results shown in Figure 7a-c indicated that compared with the IRI group (LC3-B: $1738.02 \pm 50.35 / \mathrm{mm}^{2}$; mTOR: $312.08 \pm 30.27 / \mathrm{mm}^{2}$ ), the expression level of light chain 3 (LC3)-B in the sham group $\left(1442.54 \pm 37.76 / \mathrm{mm}^{2}\right)$ decreased $(P<0.001)$, and the expression level of mammalian target of rapamycin (mTOR) $\left(494.68 \pm 23.91 / \mathrm{mm}^{2}\right)$ increased $(P<0.001)$; While, the expression level of LC3-B in the BMSCs group $\left(1163.66 \pm 21.48 / \mathrm{mm}^{2}\right)$ decreased $(\mathrm{P}<0.001)$, and the expression level of mTOR $(630.80 \pm 36.18$ 
$\left./ \mathrm{mm}^{2}\right)$ increased $(\mathrm{P}<0.001)$. Compared with the BMSCs group, the expression level of LC3-B in the OE PINK1 group $\left(1371.16 \pm 33.92 / \mathrm{mm}^{2}\right)$ increased $(P<0.001)$, and the expression level of $m T O R$ $\left(496.34 \pm 31.28 / \mathrm{mm}^{2}\right)$ decreased $(P<0.001)$, which was similar to the results in the Anti PINK1 group (LC3B: $1900.70 \pm 24.20 / \mathrm{mm}^{2}$; mTOR: 434.92 \pm 17.21$)(P<0.001 ; P<0.001)$. These results indicated that BMSCs could enhance mitophagy and repair injured tissues by increasing the expression level of LC3-B and decreasing the expression level of mTOR. PINK1 regulated mitophagy on the basis of BMSCs and better participated in the repair of IRI-AKI injuries.

\section{Discussion}

Clinical and basic trials demonstrate that BMSCs are effective in the treatment of AKI. BMSCs are recruited into injured tissues and release certain cytokines and growth factors, such as insulin-like, hepatocyte and vascular endothelial growth factors. These cytokines can activate endogenous cell repair programs, which could promote the growth and survival of endothelial cells and tubular epithelial cells, thus promoting the repair and regeneration of injured tissues [17]. However, although BMSCs have therapeutic potential, their survival rate and biological activity are relatively low under inflammatory and oxidative stress conditions at the injured site, which restricts their usage. Some studies reveal that under IRI-AKI conditions, the increased oxygen free radicals (ROSs) due to mitochondrial damage in kidney tissues are not conducive to the survival and differentiation of infused BMSCs; The pretreatment of hypoxia can improve the survival rate of BMSCs and promote the quality control of mitochondria by increasing mitophagy, thus increasing the therapeutic potential of stem cells $[18,19]$. As a key enzyme of mitophagy, PINK1 can directly participate in regulating mitophagy to improve IRI-AKI: berberine (BBR) activates mitophagy through PINK1/Parkin and reduces the accumulation of ROS to antagonize AKI induced by the nephrotoxicity of Cisplatin [20]; Pioglitazone can repair mitochondrial dysfunction induced by uremia by up-regulating PINK1 expression, inhibiting mitochondrial fusion and promoting mitophagy, thus reducing the damage degree of chronic kidney diseases (CKDs) [21]; The deletion of regulator of calcineurin 1 (RCAN1) specific to renal tubular epithelial cells could alleviate the dysfunction caused by tubulointerstitial fibrosis in CKD by regulating mitophagy induced by PINK1/Parkin [22]. The enhanced ability of therapeutic cells implanted in injured tissues to cope with such stress conditions as ischemia and hypoxia by regulating the mitophagy of PINK1/Parkin pathway could further improve IRI-AKI. Han et al. [23] proved that melatonin could up-regulate PrPC and bind it to PINK1. It could promote mitochondrial dynamics and metabolism and enhance mitochondrial function. Besides, it could protect MSCs implanted into the tissues and organs with ischemia injuries from the senescence, apoptosis and ischemia-related conditions, and promote the injury repair and tissue regeneration. This research team focuses on mitophagy of PINK1/Parkin pathway and the repair effect of BMSCs on IRI-AKI, and directly combines PINK1 with BMSCs to reduce the interference of other substances in the pathway, in an attempt to observe the repair ability of BMSCs on IRI-AKI. As can be revealed from the results, PINK1 can enhance the repair effect of BMSCs on IRI-AKI, which may be realized by the enhancing effects of PINK1 on 
BMSCs and BMSCs's resistance to stress response of RTECs under hypoxia and inflammatory conditions (shown in Fig. 4-6), and the regulatory effect of mitophagy during IRI-AKI (shown in Fig. 7). The repair mechanism of BMSCs on IRI-AKI is closely related to PINK1. The increased expression level of PINK1 may improve the activity of BMSCs implanted in the AKI microenvironment and achieve favorable therapeutic effects on AKI. Therefore, PINK1 is a potential target for the accurate treatment of IRI-AKI.

Moreover, recent studies report that renal IRI-AKI may be related to the cellular immune regulation disorder. Besides, inflammatory cytokines and immune cell infiltration play a pivotal role in kidney diseases. In addition, the extensive and complex molecular cross-reactions between renal tubular epithelial cells and immune cells, interstitial cells and endothelial cells can regulate the kidney recovery. As is revealed from a tissue biopsy, T cells or macrophages in renal parenchyma are rare under normal conditions, while inflammatory infiltration of activated T cells and macrophages during IRI-AKI exists extensively in ischemic kidneys. Further, $\mathrm{CD} 68^{+}$cells are infiltrated around glomeruli and renal tubule in different degrees $[24,25]$. Those findings are consistent with the results in the present study. Additionally, some studies also report that there is the polarity of macrophage phenotype from M1 to M2 and the transformation of phagocytic function to structural repair function, combined with the formation of new capillaries and the regeneration of renal tubules; It is worth noting that BMSCs can modify inflammatory cells under ischemia and hypoxia conditions to facilitate the transformation of immunophenotype [26]. BMSCs would present immunosuppressive effects only when they are exposed to a high enough level of pro-inflammatory cytokines, which can promote tissue repair and inhibit the occurrence of inflammatory reaction [27]. As is highlighted in abundant studies, the immunomodulatory characteristics of BMSCs are closely related to the immune response mediated by $T$ cells, B cells and NK cells. In addition, BMSCs could participate in the immune response by regulating the activation, expansion and transformation of the expression profile of proteins that play an important role in their immune function [28, 29]. The important role of mitochondria in regulating the dynamic network of congenital and acquired immune signaling pathways has been emphasized in recent studies; As a key enzyme to maintain mitochondrial homeostasis, PINK1 participates in the occurrence and development of many diseases, such as IRI [30, 31]. Mitophagy mediated by PINK1 can eliminate damaged mitochondria, maintain the microenvironmental homeostasis and inhibit the occurrence of the immune response. As per the results of this study, PINK1 decreases the infiltration of lymphocytes and B cells and increases the infiltration of macrophages and monocytes during the treatment with BMSCs (shown in Fig. 3). It indicates that PINK1 could enhance the ability of BMSCs to inhibit the immune response by regulating the distribution of immune cells in the damaged kidney during IR. PINK1 and BMSCs can synergistically regulate and alleviate the immune disorder in kidneys with IRI-AKI, and enhance the Anti-inflammatory and immunosuppressive properties of BMSCs, thus promoting the repair of kidneys with IRI-AKI.

However, there are still obstacles to the application of stem cell therapy in the clinical environment, such as side effects, high cost, difficulties in controlling the optimal infusion time and the selection of the optimal infusion approach [14]. Besides, it can also be found that BMSCs can damage the lung and liver to a certain extent during the repair of the kidney of mice, which may be caused by cell embolism. 
According to a study with respect to a rodent kidney injury model, after the intravenous injection of BMSCs, most cells are trapped in both lungs due to the pulmonary first-pass elimination, which would cause partial damage to lung tissues [32]. In addition, a meta-analysis also reveal that it is difficult to avoid lung tissue injury resulting from the blockage of BMSCs by both lungs due to the kidney injury caused by different methods, injection of BMSCs at different time points after injury and different approaches of the injection of BMSCs [33]. It can be observed that BMSCs with overexpressed PINK1 can mitigate lung injury and may promote the entry of BMSCs into damaged organs. Further, it can be found that BMSCs still have a favorable repair effect on damaged tissues after passing through the pulmonary first-pass elimination. This effect may be related to the close relationship between the repair mechanism of BMSCs and its paracrine function. Moreover, PINK1 can enhance the paracrine function of BMSCs. BMSCs with overexpressed PINK1 have enhanced ability to promote the repair of kidney tissues damaged by IRI-AKI. Besides, they can secrete abundant cytokines and growth factors to promote immunosuppression, Anti-inflammation, Anti-apoptosis and proliferation, which would promote the recovery of renal function. As a cell transplantation scheme, BMSCs with overexpressed PINK1 have significant therapeutic potential, therapeutic attraction and clinical application value for the treatment of IRI-AKI. There are also practical problems for the application of BMSCs with overexpressed PINK1 as a treatment method. For example, the method to reduce the pulmonary first-pass elimination without reducing its specific curative effect on IRI-AKI, the content of PINK1 carried by BMSCs, and the verification of the more accurate targeting of treatment are the challenges that shall be overcome in the future.

\section{Conclusion}

In this study, PINK1 overexpression enhances the repair effect of BMSCs on IRI-AKI, and the distribution of injured renal immune cells during IRI regulation by BMSCs. Besides, PINK1 enhances BMSCs and their resistance to the stress response of RTECs under hypoxia and inflammation. In addition, it regulates mitophagy during IRI-AKI. The findings of this study provide a new direction and target for the repair of IRI-AKI through BMSCs.

\section{Abbreviations}

AKI

Acute kidney injury

BMSCs

Bone mesenchymal stem cells

PINK1

PENT-induced kinase 1

AKI

acute kidney injury

IRI

ischemia/reperfusion injury 
IRI-AKI

the ischemia/reperfusion-induced acute kidney injury

RTECs

renal tubular epithelial cells

LC3

light chain 3

mTOR

mammalian target of rapamycin

ROSs

reactive oxygen species

SCR

serum creatinine

BUN

blood urea nitrogen

HE

hematoxylin-eosin

TUNEL

Terminal deoxynucleotidyl transferase dUTP nick end labeling

FCM

Flow Cytometry

IL-10

interleukin-10

TNF-a

tumor necrosis factor-a

PBMCs

peripheral blood mononuclear cells.

\section{Declarations}

\section{Ethics approval and consent to participate}

All animal experiments were approved by the Ethics Committee of Laboratory Animal Management in the 8th Medical Center of Chinese PLA General Hospital (309202106021005) ; the animal experiments conform to internationally accepted standards.

\section{Consent for publication}

Not applicable.

\section{Availability of data and materials}


The datasets used and/or analyzed during the current study are available from the corresponding author on reasonable request.

\section{Competing interests}

The authors declare that they have no competing interests.

\section{Funding}

This work was supported by National Science Foundation of China Grants 81571555 (Xiao L.) and military medical science and technology youth training top-notch project 18QNP035 (Xiao L.).

\section{Author Contributions}

LC completed preliminary research, conducted experiments, analyzed data and wrote original draft manuscripts. CW designed the experiment and revised and corrected the manuscript, HY completed pathology related experiments, SY and LB conducted flow cytometry detection and analysis, $Z X$ and $Y Y$ took pictures of the experiment, FW constructed the plasmid corrected and revised the manuscript, $\mathrm{ZT}$ corrected and revised the manuscript, $\mathrm{XL}$ designed the experiment, managed the project, and supervised the experiment progress.

\section{Acknowledgements}

Not applicable.

\section{References}

1. Moore PK, Hsu RK, Liu KD. Management of Acute Kidney Injury: Core Curriculum 2018. Am J Kidney Dis. 2018 Jul;72(1):136-48. doi:10.1053/j.ajkd.2017.11.021. Epub 2018 Feb 22. PMID: 29478864.

2. Quenot JP, Dargent A, Large A, Roudaut JB, Andreu P, Barbar S. Treatment of sepsis-induced acute kidney injury in the ICU: the therapeutic targets do not seem to be established yet. Ann Transl Med. 2019 Sep;7(Suppl 6):181. doi:10.21037/atm.2019.07.66. PMID: 31656760; PMCID: PMC6789372.

3. Venkatachalam MA, Weinberg JM, Kriz W, Bidani AK. Failed Tubule Recovery AKI-CKD. Transition, and Kidney Disease Progression. J Am Soc Nephrol. 2015 Aug;26(8):1765-76. doi:10.1681/ASN.2015010006. Epub 2015 Mar 25. PMID: 25810494; PMCID: PMC4520181.

4. Havasi A, Dong Z. Autophagy and Tubular Cell Death in the Kidney. Semin Nephrol. 2016 May;36(3):174-88. doi:10.1016/j.semnephrol.2016.03.005. PMID: 27339383; PMCID: PMC4920968. 
5. Livingston MJ, Wang J, Zhou J, Wu G, Ganley IG, Hill JA, et al. Clearance of damaged mitochondria via mitophagy is important to the protective effect of ischemic preconditioning in kidneys. Autophagy. 2019 Dec;15(12):2142-62. doi:10.1080/15548627.2019.1615822. Epub 2019 May 22. PMID: 31066324; PMCID: PMC6844514.

6. Zhang Y, Whaley-Connell AT, Sowers JR, Ren J. Autophagy as an emerging target in cardiorenal metabolic disease: From pathophysiology to management. Pharmacol Ther. 2018 Nov;191:1-22. doi:10.1016/j.pharmthera.2018.06.004. Epub 2018 Jun 22. PMID: 29909238; PMCID: PMC6195437.

7. Wang Y, Zhu J, Liu Z, Shu S, Fu Y, Liu Y, et al. The PINK1/PARK2/optineurin pathway of mitophagy is activated for protection in septic acute kidney injury. Redox Biol. 2021 Jan;38:101767. doi: 10.1016/j.redox.2020.101767. Epub 2020 Oct 23. PMID: 33137712; PMCID: PMC7606859.

8. Zhang HT, Mi L, Wang T, Yuan L, Li XH, Dong LS, et al. PINK1/Parkin-mediated mitophagy play a protective role in manganese induced apoptosis in SH-SY5Y cells. Toxicol In Vitro. 2016 Aug;34:2129. doi:10.1016/j.tiv.2016.04.006. Epub 2016 Apr 16. PMID: 27091500.

9. Lazarou M, Sliter DA, Kane LA, Sarraf SA, Wang C, Burman JL, et al. The ubiquitin kinase PINK1 recruits autophagy receptors to induce mitophagy. Nature. 2015 Aug 20;524(7565):309-314. doi: 10.1038/nature14893. Epub 2015 Aug 12. PMID: 26266977; PMCID: PMC5018156.

10. Ahmadi A, Rad NK, Ezzatizadeh V, Moghadasali R. Kidney Regeneration: Stem Cells as a New Trend. Curr Stem Cell Res Ther. 2020;15(3):263-283. doi: 10.2174/1574888X15666191218094513. PMID: 31854277.

11. Textor SC, Abumoawad A, Saad A, Ferguson C, Dietz A. Stem Cell Therapy for Microvascular Injury Associated with Ischemic Nephropathy. Cells. 2021 Mar 31;10(4):765. doi: 10.3390/cells10040765. PMID: 33807289; PMCID: PMC8066553.

12. Monsel A, Zhu YG, Gennai S, Hao Q, Liu J, Lee JW. Cell-based therapy for acute organ injury: preclinical evidence and ongoing clinical trials using mesenchymal stem cells. Anesthesiology. 2014 Nov;121(5):1099-121. doi:10.1097/ALN.0000000000000446. PMID: 25211170; PMCID: PMC4206665.

13. Xu X, Duan S, Yi F, Ocampo A, Liu GH, Izpisua Belmonte JC. Mitochondrial regulation in pluripotent stem cells. Cell Metab. 2013 Sep 3;18(3):325-32. doi: 10.1016/j.cmet.2013.06.005. Epub 2013 Jul 11. PMID: 23850316.

14. Yun CW, Lee SH. Potential and Therapeutic Efficacy of Cell-based Therapy Using Mesenchymal Stem Cells for Acute/chronic Kidney Disease. Int J Mol Sci. 2019 Apr 1;20(7):1619. doi: 10.3390/ijms20071619. PMID: 30939749; PMCID: PMC6479813.

15. Wang J, Zhu P, Li R, Ren J, Zhou H. Fundc1-dependent mitophagy is obligatory to ischemic preconditioning-conferred renoprotection in ischemic AKI via suppression of Drp1-mediated mitochondrial fission. Redox Biol. 2020 Feb;30:101415. doi:10.1016/j.redox.2019.101415. Epub 2019 Dec 28. PMID: 31901590; PMCID: PMC6940662.

16. Henry P, Popescu A, Pucéat M, Hinescu ME, Escande D. Acute simulated ischaemia produces both inhibition and activation of K+ currents in isolated ventricular myocytes. Cardiovasc Res. 1996 
Nov;32(5):930-9. PMID: 8944824.

17. Jia H, Yan Y, Liang Z, Tandra N, Zhang B, Wang J, et al. Autophagy: A new treatment strategy for MSC-based therapy in acute kidney injury (Review). Mol Med Rep. 2018 Mar;17(3):3439-3447. doi: 10.3892/mmr.2017.8311. Epub 2017 Dec 19. PMID: 29257336.

18. Liu N, Wang H, Han G, Cheng J, Hu W, Zhang J. Enhanced proliferation and differentiation of HO-1 gene-modified bone marrow-derived mesenchymal stem cells in the acute injured kidney. Int J Mol Med. 2018 Aug;42(2):946-56. doi:10.3892/ijmm.2018.3670. Epub 2018 May 11. PMID: 29749549; PMCID: PMC6034926.

19. Zhang W, Liu L, Huo Y, Yang Y, Wang Y. Hypoxia-pretreated human MSCs attenuate acute kidney injury through enhanced angiogenic and Antioxidative capacities. Biomed Res Int. 2014;2014:462472. doi:10.1155/2014/462472. Epub 2014 Jul 16. PMID: 25133162; PMCID: PMC4123714.

20. Qi J, Xue Q, Kuang L, Xie L, Luo R, Nie X. Berberine alleviates cisplatin-induced acute kidney injury by regulating mitophagy via PINK 1/Parkin pathway. Transl Androl Urol. 2020 Aug;9(4):1712-24. doi:10.21037/tau-20-1129. PMID: 32944532; PMCID: PMC7475663.

21. Yoon YM, Han YS, Yun CW, Lee JH, Kim R, Lee SH. Pioglitazone Protects Mesenchymal Stem Cells against P-Cresol-Induced Mitochondrial Dysfunction via Up-Regulation of PINK-1. Int J Mol Sci. 2018 Sep 24;19(10):2898. doi: 10.3390/ijms19102898. PMID: 30250007; PMCID: PMC6213327.

22. Sang XY, Xiao JJ, Liu Q, Zhu R, Dai JJ, Zhang C, et al. Regulators of calcineurin 1 deficiency attenuates tubulointerstitial fibrosis through improving mitochondrial fitness. FASEB J 2020 Nov;34(11). doi:10.1096/fj.202000781RRR. Epub 2020 Sep 7. PMID: 32896034.

23. Han YS, Kim SM, Lee JH, Jung SK, Noh H, Lee SH. Melatonin protects chronic kidney disease mesenchymal stem cells against senescence via PrPC -dependent enhancement of the mitochondrial function. J Pineal Res. 2019 Jan;66(1):e12535. doi: 10.1111/jpi.12535. Epub 2018 Nov 18. Erratum in: J Pineal Res. 2020 May;68(4):e12651. PMID: 30372554.

24. Kumar S. Cellular and molecular pathways of renal repair after acute kidney injury. Kidney Int. 2018 Jan;93(1):27-40. doi: 10.1016/j.kint.2017.07.030. PMID: 29291820.

25. Xie X, Yang X, Wu J, Ma J, Wei W, Fei X, et al. Trib1 Contributes to Recovery From Ischemia/Reperfusion-Induced Acute Kidney Injury by Regulating the Polarization of Renal Macrophages. Front Immunol. 2020 Mar 20;11:473. doi: 10.3389/fimmu.2020.00473. PMID: 32265926; PMCID: PMC7098949.

26. Puranik AS, Leaf IA, Jensen MA, Hedayat AF, Saad A, Kim KW, et al. Kidney-resident macrophages promote a proangiogenic environment in the normal and chronically ischemic mouse kidney. Sci Rep. 2018 Sep 17;8(1):13948. doi: 10.1038/s41598-018-31887-4. PMID: 30224726; PMCID: PMC6141464.

27. Anegon I, Nguyen TH. 21st Nantes Actualités Transplantation: "When Stem Cells Meet Immunology". Transplantation. 2017 Jan;101(1):12-16. doi: 10.1097/TP.0000000000001476. PMID: 27653295. 
28. Ribeiro A, Laranjeira P, Mendes S, Velada I, Leite C, Andrade P, et al. Mesenchymal stem cells from umbilical cord matrix, adipose tissue and bone marrow exhibit different capability to suppress peripheral blood B, natural killer and T cells. Stem Cell Res Ther. 2013 Oct 15;4(5):125. doi: 10.1186/scrt336. PMID: 24406104; PMCID: PMC3854702.

29. Dorronsoro A, Ferrin I, Salcedo JM, Jakobsson E, Fernández-Rueda J, Lang V, et al. Human mesenchymal stromal cells modulate T-cell responses through TNF-a-mediated activation of NF-KB. Eur J Immunol. 2014 Feb;44(2):480-8. doi: 10.1002/ eji.201343668. Epub 2013 Dec 4. PMID: 24307058.

30. Sliter DA, Martinez J, Hao L, Chen X, Sun N, Fischer TD, et al. Parkin and PINK1 mitigate STINGinduced inflammation. Nature. 2018 Sep;561(7722):258-62. doi:10.1038/s41586-018-0448-9. Epub 2018 Aug 22. PMID: 30135585; PMCID: PMC7362342.

31. Tiku V, Tan MW, Dikic I. Mitochondrial Functions in Infection and Immunity. Trends Cell Biol. 2020 Apr;30(4):263-275. doi: 10.1016/j.tcb.2020.01.006. Epub 2020 Feb 11. Erratum in: Trends Cell Biol. 2020 Sep;30(9):748. PMID: 32200805; PMCID: PMC7126537.

32. Schrepfer S, Deuse T, Reichenspurner H, Fischbein MP, Robbins RC, Pelletier MP. Stem cell transplantation: the lung barrier. Transplant Proc. 2007 Mar;39(2):573-6. doi: 10.1016/j.transproceed.2006.12.019. PMID: 17362785.

33. Taylor A, Sharkey J, Harwood R, Scarfe L, Barrow M, Rosseinsky MJ, et al. Multimodal Imaging Techniques Show Differences in Homing Capacity Between Mesenchymal Stromal Cells and Macrophages in Mouse Renal Injury Models. Mol Imaging Biol. 2020 Aug;22(4):904-913. doi: 10.1007/s11307-019-01458-8. PMID: 31823201; PMCID: PMC7343735.

\section{Figures}




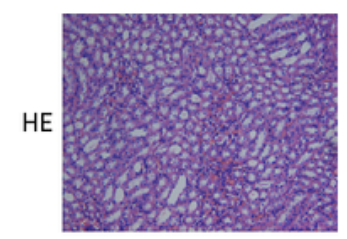

Sham

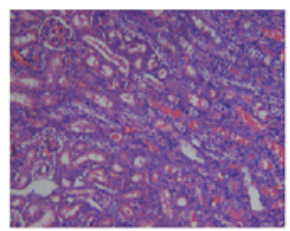

|R|

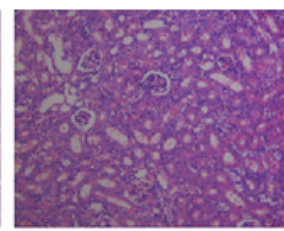

BMSCs

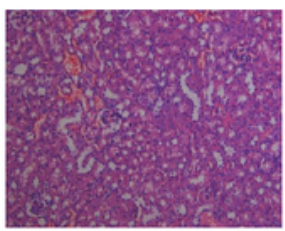

OE PINK1

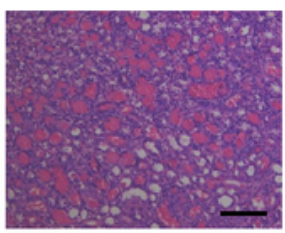

Anti PINK1
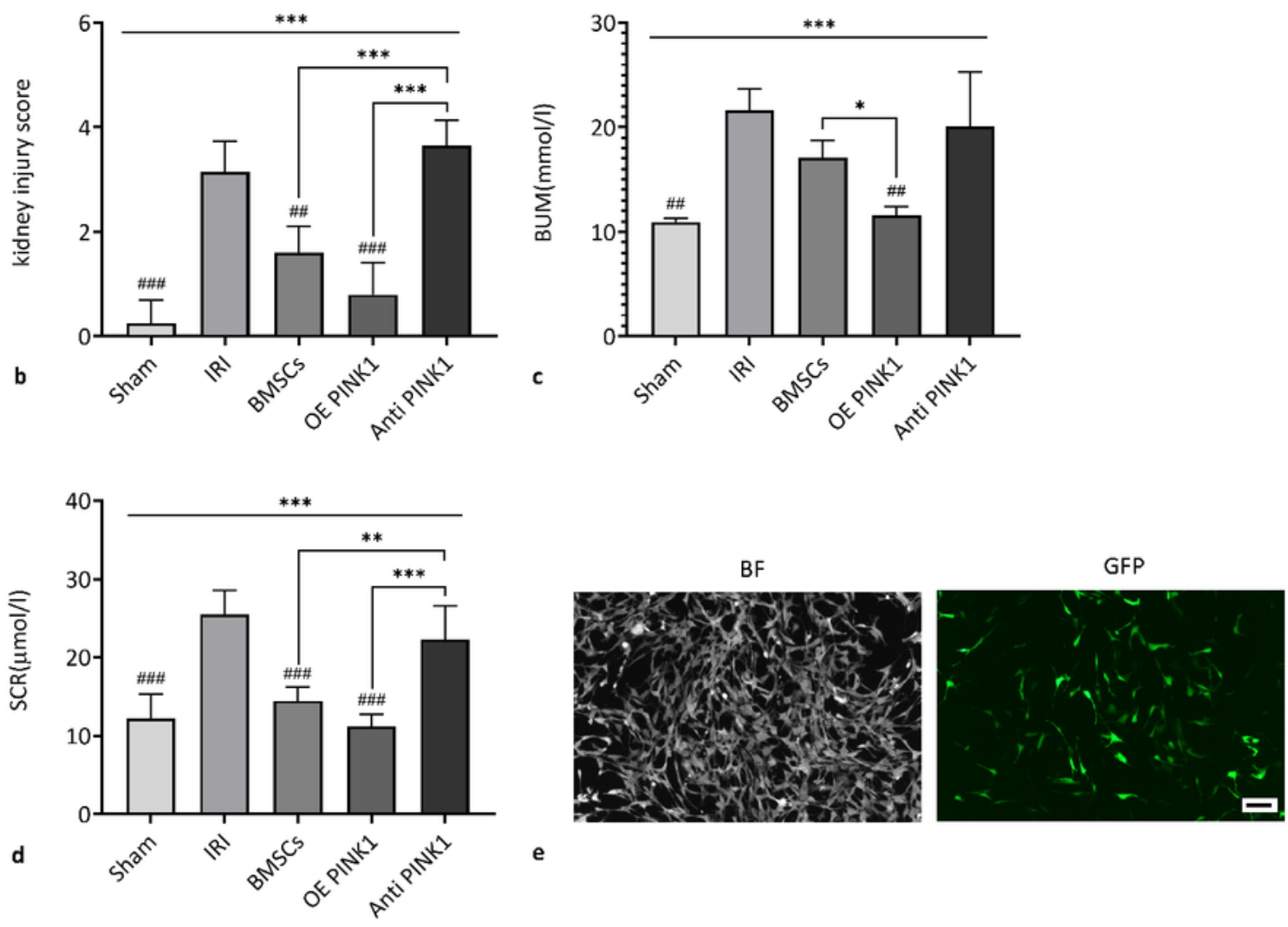

RFP

DAPI

Merge
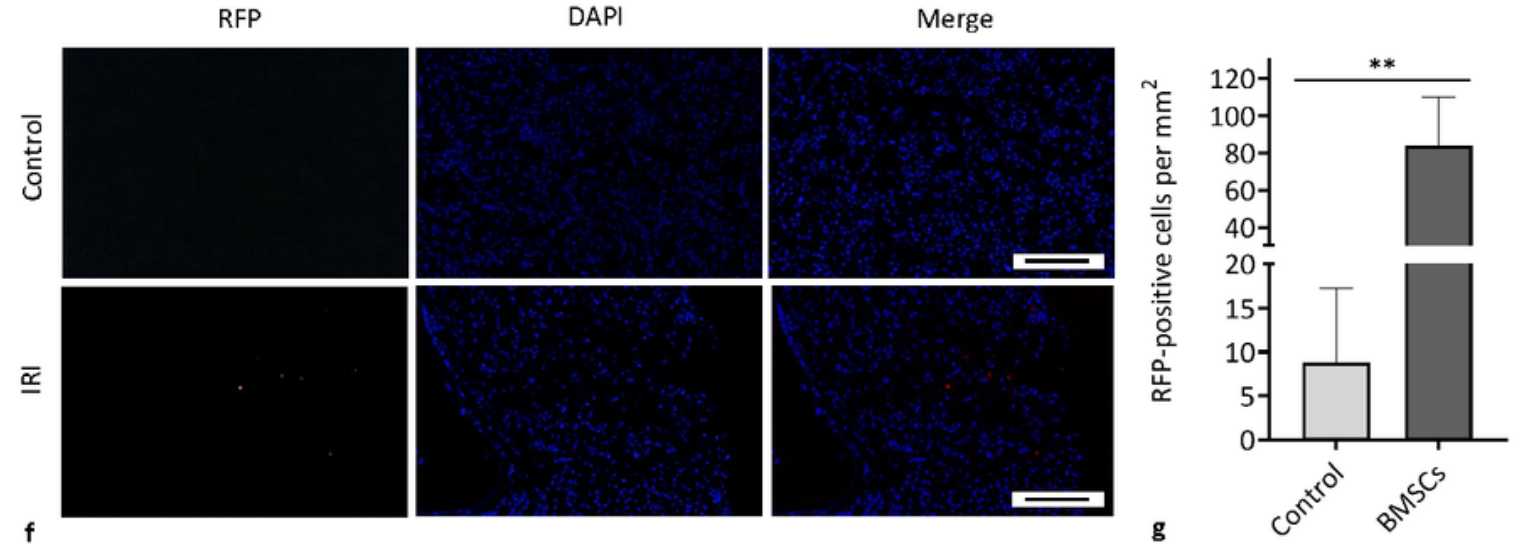

Figure 1

PINK1 enhances the repair effect of BMSCs on kidney tissues of IRI-AKI mice. a: Representative image of HE staining, $x 200$, scale: $100 \mu \mathrm{m}$. b: Pathological score of renal tubular injury $(n=6)$. c: The determination of BUN through blood sample collection $(n=6)$. d: The determination of SCR through blood sample

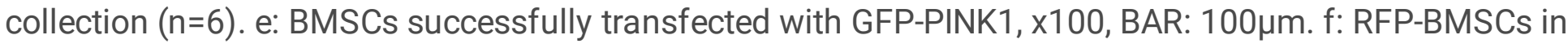

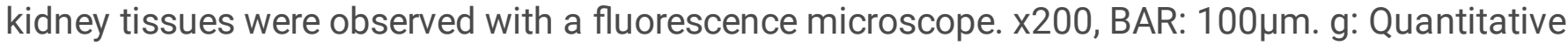




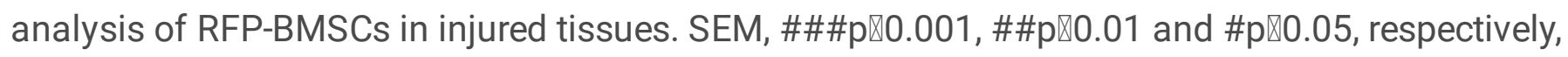
compared with the IRI group; ${ }^{* \star *} p<0.001,{ }^{* *} p<0.01$ and ${ }^{*} p<0.05$, respectively, compared with among groups.
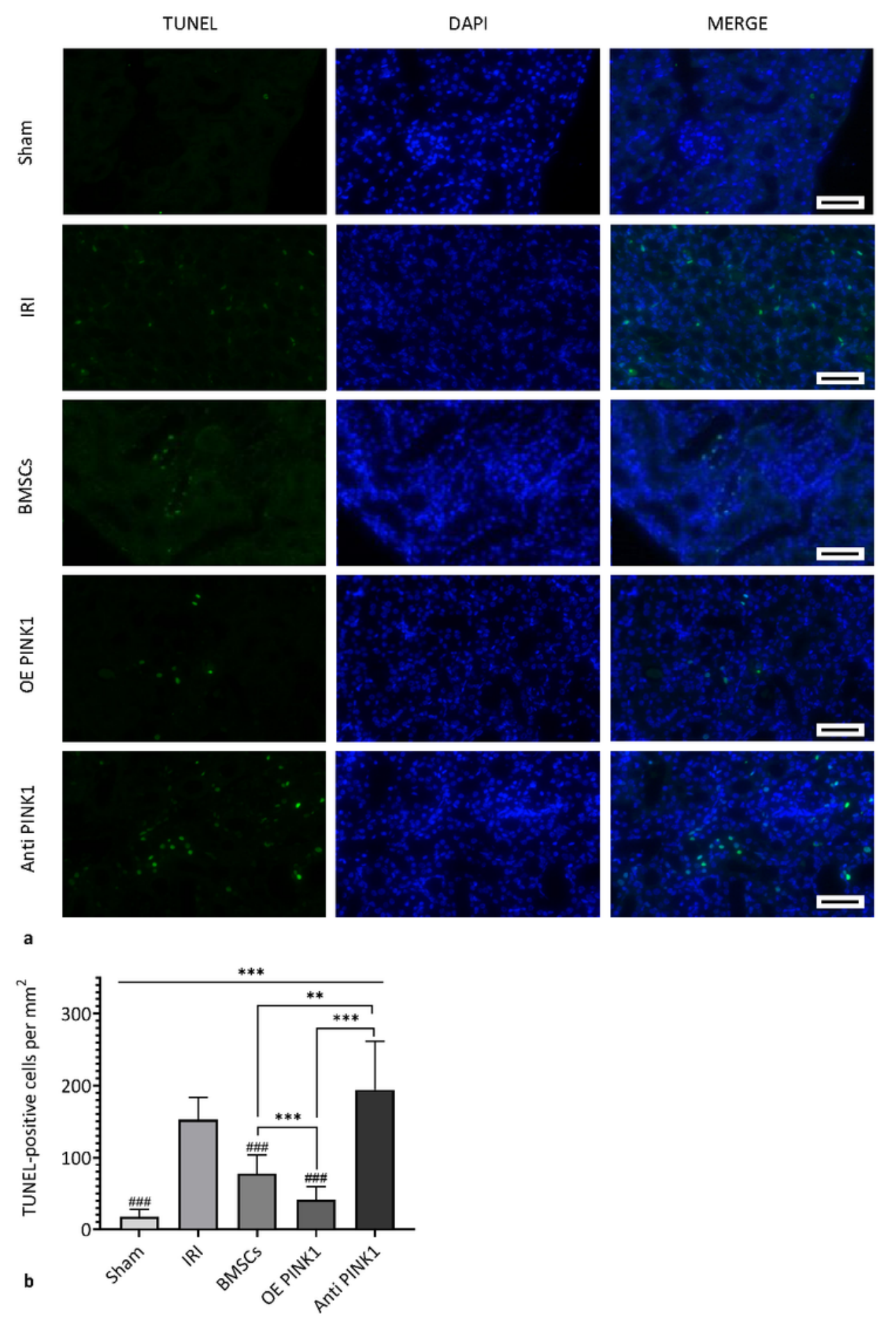

Figure 2 
PINK1 enhances the inhibiting effect of BMSCs on apoptosis in kidney tissues of IRI-AKI mice. a:

Representative image of TUNEL staining in kidney tissues. b: The number of TUNEL-positive cells in each

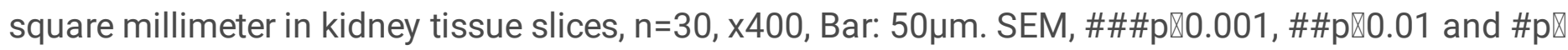
0.05 , respectively, compared with the IRI group; ${ }^{* \star} p<0.001,{ }^{* *} p<0.01$ and ${ }^{*} p<0.05$, respectively, compared with among groups.
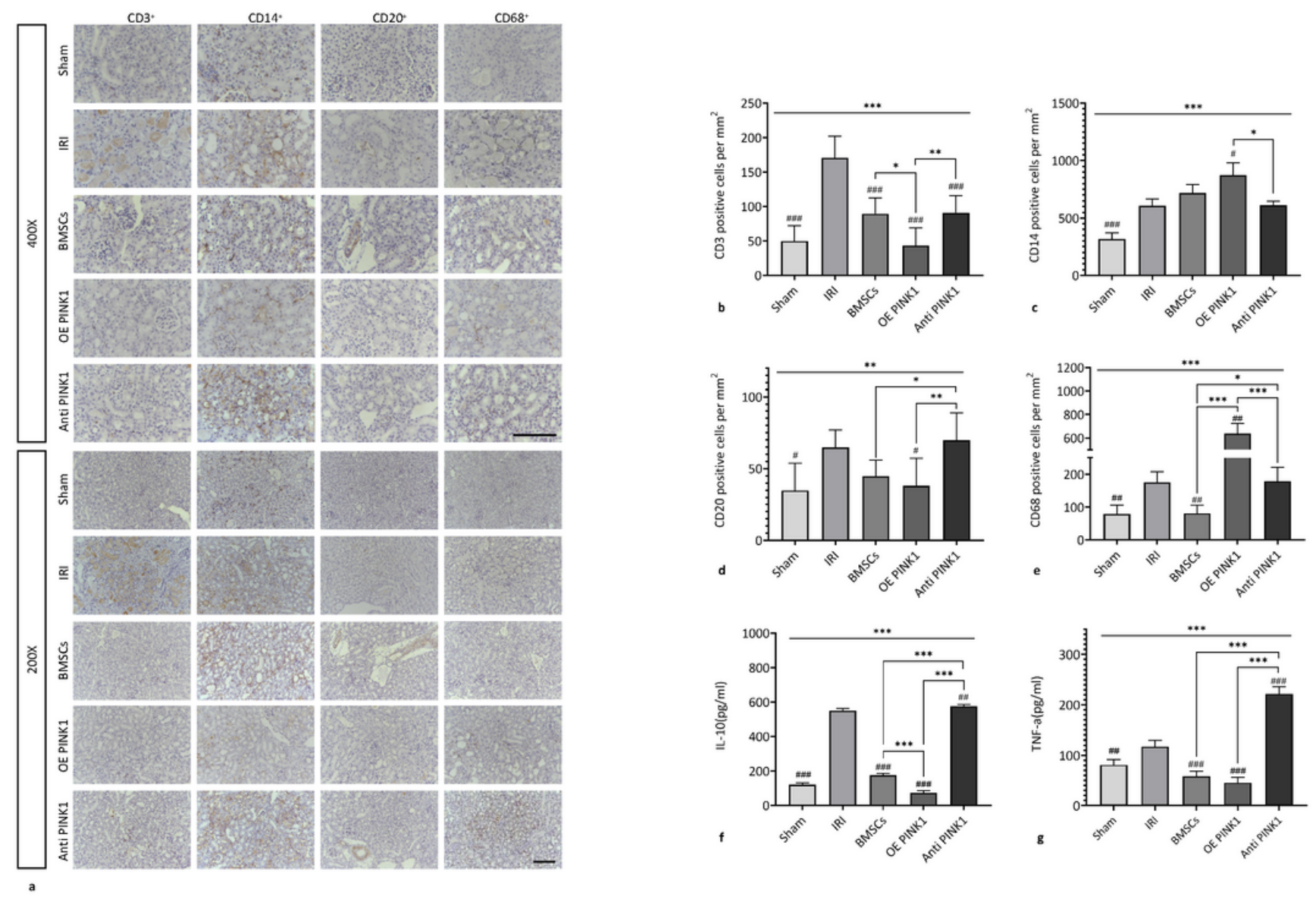

\section{Figure 3}

PINK1 enhances the Anti-inflammatory effect of BMSCs on the kidney of IRI-AKI mice. a: Representative image of immunohistochemical staining. $x 400$ and $x 200$, respectively, BAR: $100 \mu \mathrm{m}$. b: CD3 positive cell counting $(n=6)$. c: CD14 positive cell counting $(n=6)$. d: CD20 positive cell counting $(n=6)$. e: CD68 positive cell counting $(n=6)$. f: Relative changes in the expression of IL-10 in peripheral blood $(n=6)$. g: Relative changes in the expression of tumor necrosis factor-a in peripheral blood $(n=6)$. SEM, \#\#\#p》 $0.001, \# \# p \otimes 0.01$ and $\# p \otimes 0.05$, respectively, compared with the IRI group; ${ }^{* \star} \mathrm{p}<0.001,{ }^{* \star} \mathrm{p}<0.01$ and ${ }^{*} \mathrm{p}<0.05$, respectively, compared with among groups. 


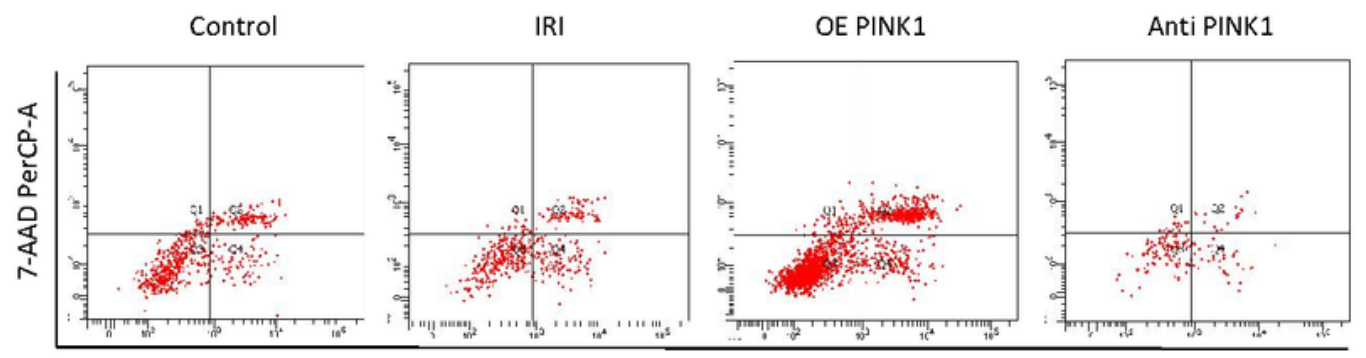

a

AnnexinV PE-A
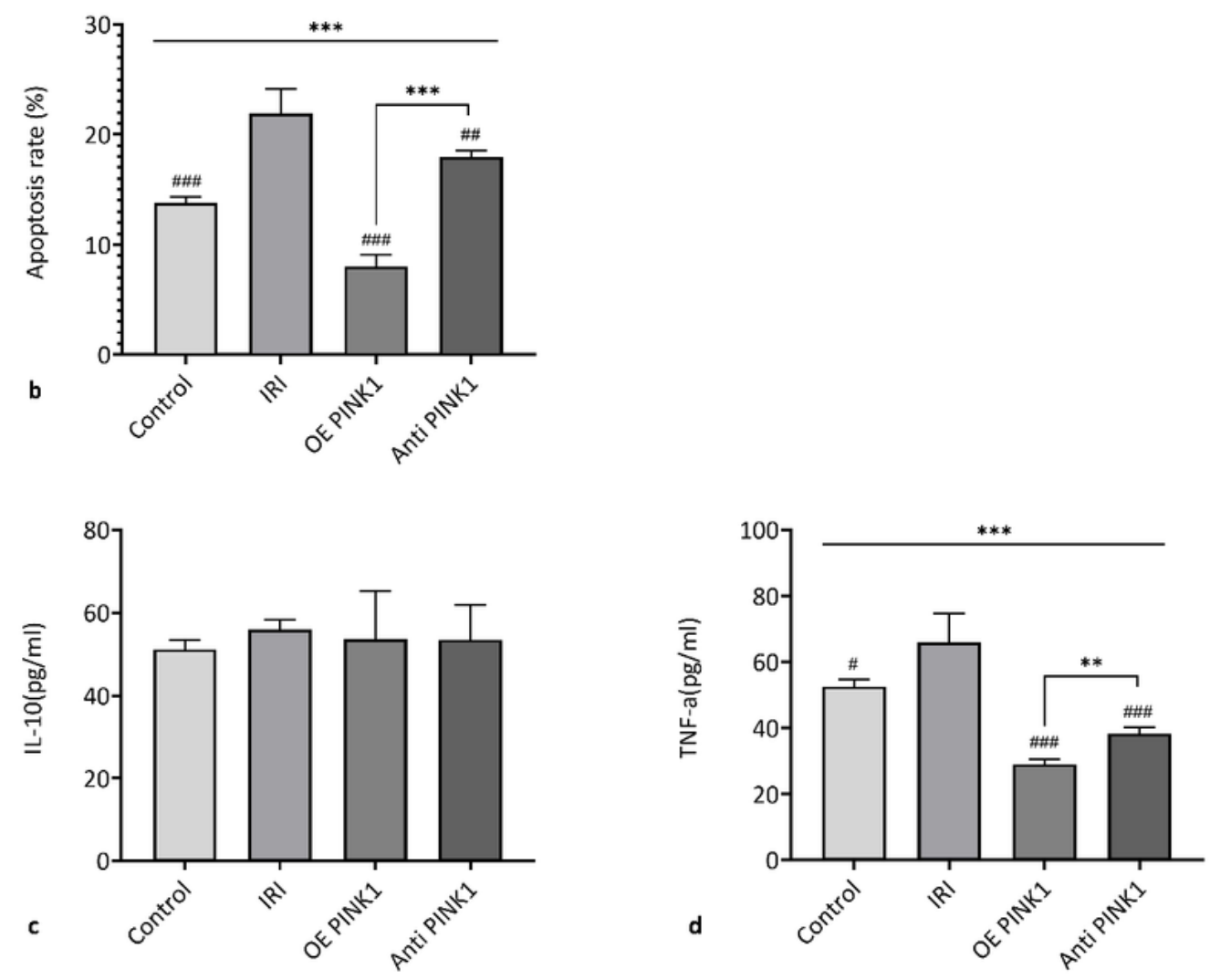

Figure 4

PINK1 mitigates the stress response of BMSCs under hypoxia-reoxygenation conditions. a:

Representative image of the apoptosis of BMSCs detected by flow cytometry. b: Quantitative analysis of apoptosis detection ( $n=3)$. c: Relative changes in the expression level of IL-10 in cell supernatant $(n=3)$. d:

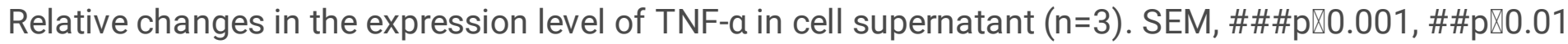


and \#p $₫ 0.05$, respectively, compared with the IRI group; ${ }^{\star \star *} p<0.001,{ }^{*} p<0.01$ and ${ }^{*} p<0.05$, respectively, compared with among groups.
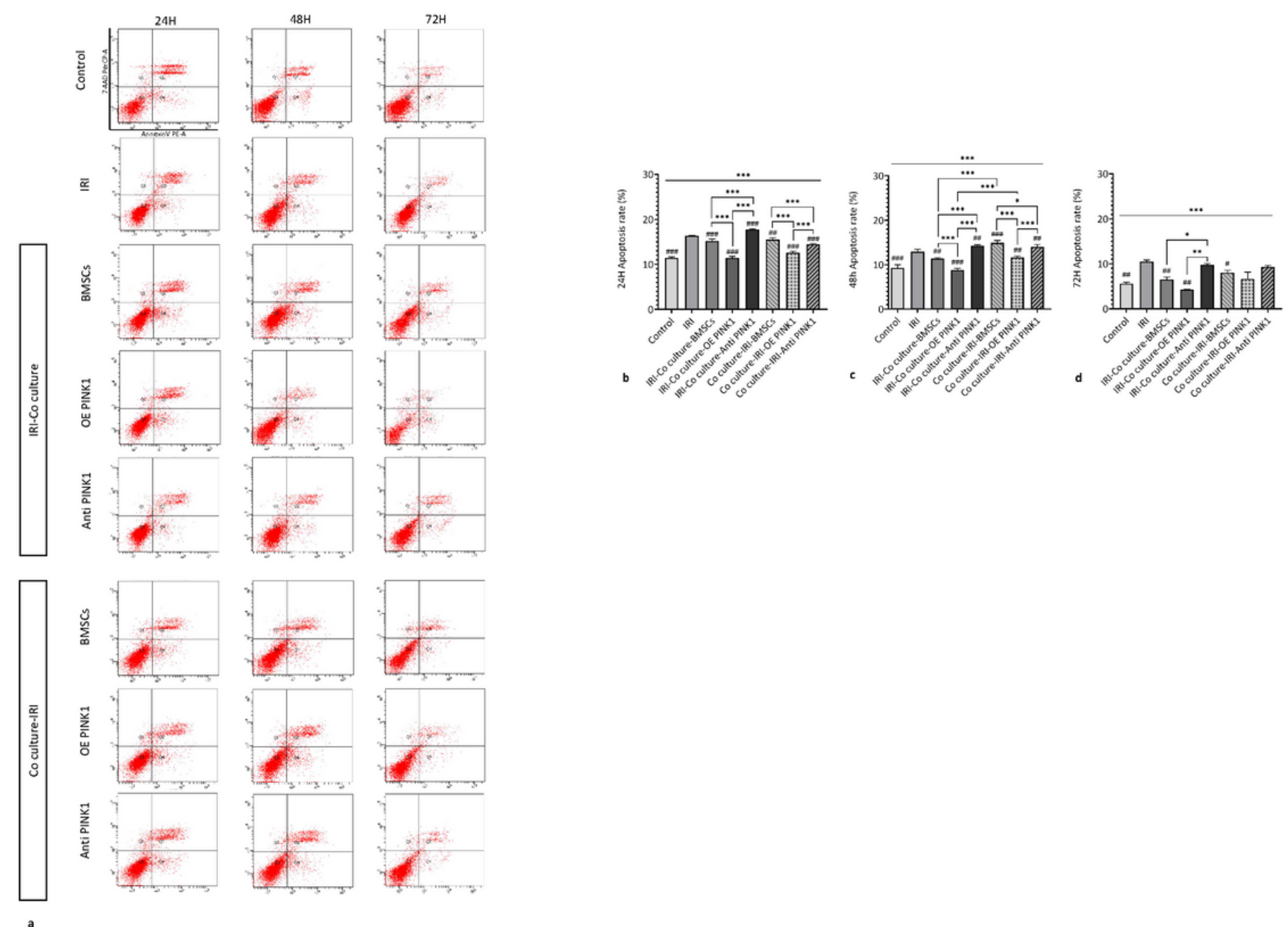

\section{Figure 5}

PINK1 enhances the effect of BMSCs on reducing apoptosis of RTECs under hypoxia-reoxygenation conditions. a: Representative image of apoptosis detected by FCM. b: Quantitative analysis results of $24 \mathrm{H}$ apoptosis rate. c: Quantitative analysis results of $48 \mathrm{H}$ apoptosis rate. d: Quantitative analysis results

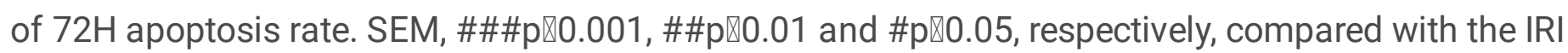
group; $* \star * p<0.001, * * p<0.01$ and $* p<0.05$, respectively, compared with among groups. 


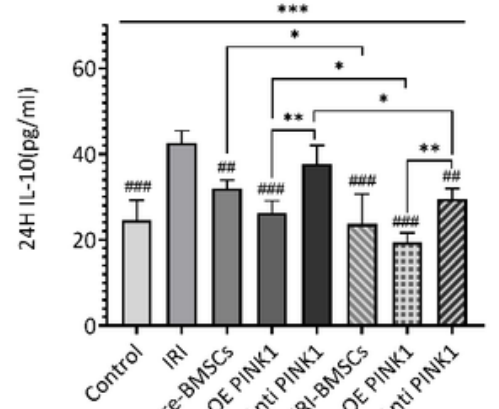

a
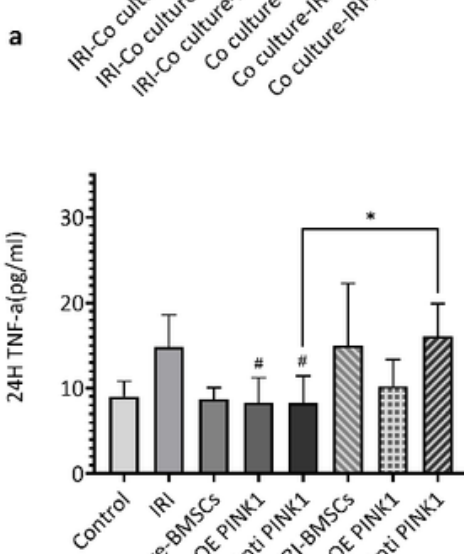

d

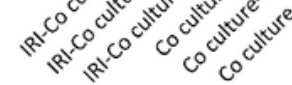

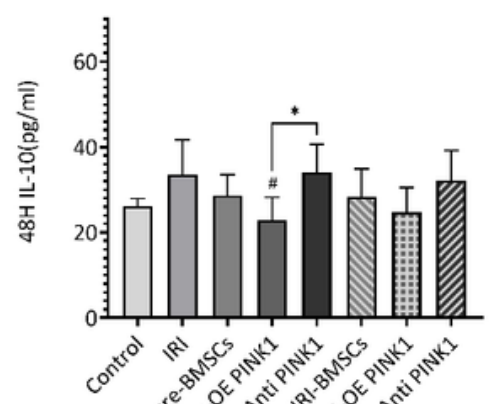

b
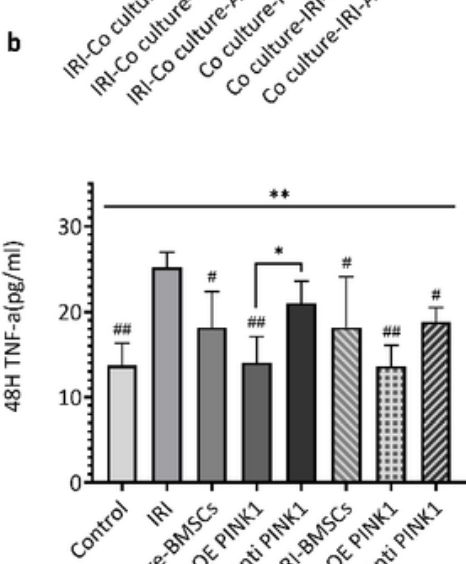

e

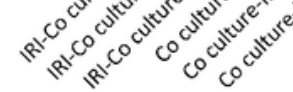

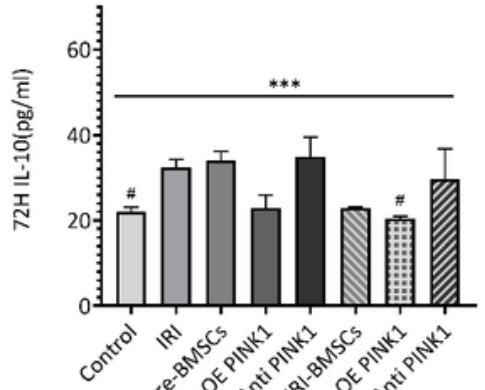

c
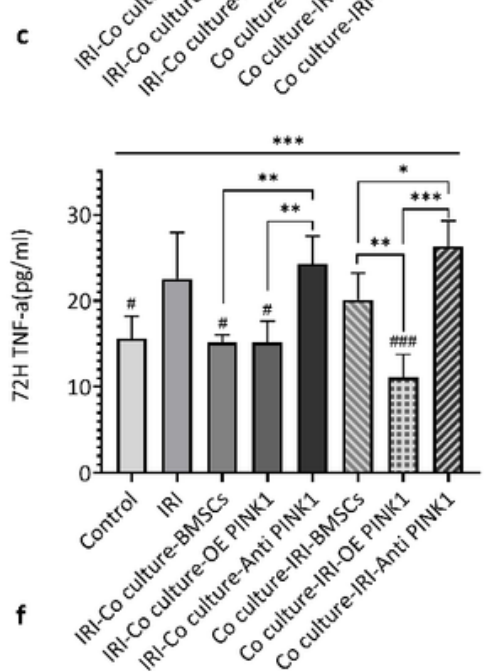
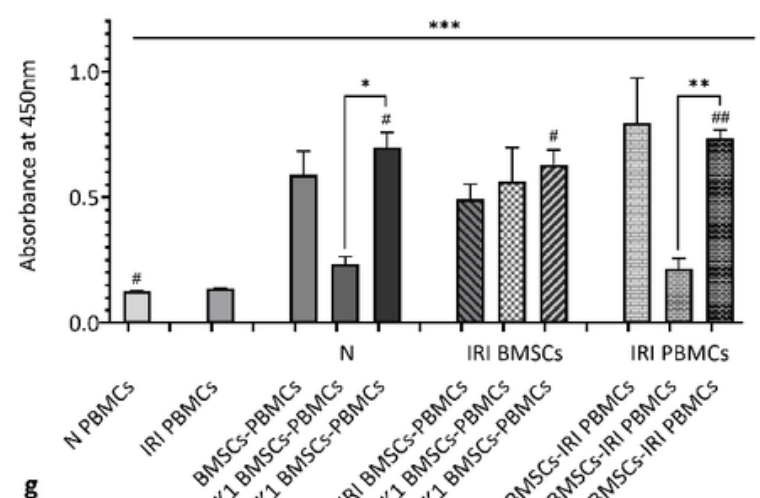

\section{Figure 6}

PINK1 enhances the release of inflammatory factors from co-cultured BMSCs-RTECs under hypoxiareoxygenation conditions. $a, b$ and $c$ : The expression level of IL-10 in the co-cultured supernatant after 24h, 48h and 72h, respectively. $\mathrm{d}$, e and f: The expression level of TNF-a in the co-cultured supernatant after $24 \mathrm{~h}, 48 \mathrm{~h}$ and $72 \mathrm{~h}$, respectively. g: Absorbance analysis related to the proliferation of PBMCs 
detected by CCK8. SEM, \#\#\#p $₫ 0.001, \# \# \mathrm{p} \varangle 0.01$ and \#p $₫ 0.05$, respectively, compared with the IRI group; ${ }^{\star * *} \mathrm{p}<0.001,{ }^{* \star} \mathrm{p}<0.01$ and ${ }^{*} \mathrm{p}<0.05$, respectively, compared with among groups.
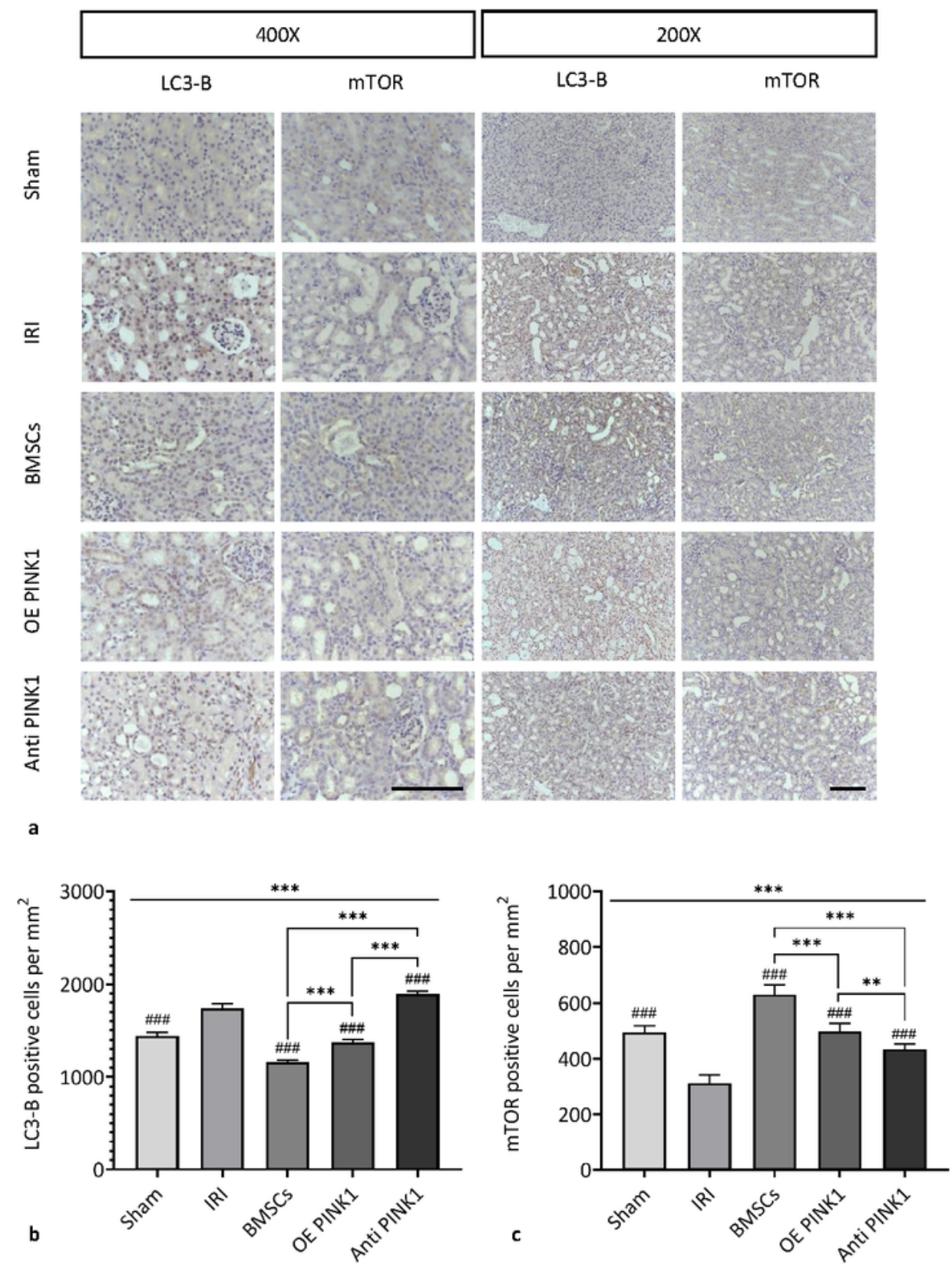

Figure 7

PINK1 participates in IRI-AKI through targeting mitophagy. a: Representative image of LC3-B and mTOR through immunohistochemical staining in kidney tissues. $x 400$ and $x 200$, respectively, BAR: $100 \mu \mathrm{m}$. b: 
The number of positive cells for LC3-B through immunohistochemical staining. c: The number of positive

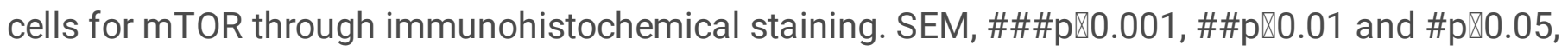
respectively, compared with the IRI group; ${ }^{* \star} \mathrm{p}<0.001,{ }^{*} \mathrm{p}<0.01$ and ${ }^{*} \mathrm{p}<0.05$, respectively, compared with among groups. 